OPEN ACCESS

Edited by:

Guigen $L i$,

Texas Tech University, United States

Reviewed by:

Huayue Wu,

Wenzhou University, China

Aamer Saeed,

Quaid-i-Azam University, Pakistan

${ }^{*}$ Correspondence: Yongmin Ma

yongmin.ma@tzc.edu.cn

In Memoriam:

This paper is dedicated to the memory of Prof. Yongmin Zhang (1932-2019).

Specialty section:

This article was submitted to

Organic Chemistry,

a section of the journal

Frontiers in Chemistry

Received: 21 June 2019 Accepted: 02 August 2019 Published: 16 August 2019

Citation:

Yan H, Xiao X-Q, Hider RC and Ma Y (2019) A Simple Metal-Free

Cyclization for the Synthesis of

4-Methylene-3-Substituted

Quinazolinone and Quinazolinthione

Derivatives: Experiment and Theory.

Front. Chem. 7:584.

doi: 10.3389/fchem.2019.00584

\section{A Simple Metal-Free Cyclization for the Synthesis of 4-Methylene-3-Substituted Quinazolinone and Quinazolinthione Derivatives: Experiment and Theory}

\author{
Huihui Yan ${ }^{1,2}$, Xu-Qiong Xiao ${ }^{3}$, Robert C. Hider ${ }^{4}$ and Yongmin Ma ${ }^{1,2 *}$ \\ ${ }^{1}$ School of Pharmaceutical and Chemical Engineering, Taizhou University, Taizhou, China, ${ }^{2}$ School of Pharmaceutical \\ Science, Zhejiang Chinese Medical University, Hangzhou, China, ${ }^{3}$ Key Laboratory of Organosilicon Chemistry and Material \\ Technology of Ministry of Education, Hangzhou Normal University, Hangzhou, China, ${ }^{4}$ Institute of Pharmaceutical Science, \\ King's College London, London, United Kingdom
}

A new series of 3-substituted 4-methylene-quinazolinthiones and 4-methylenequinazolinones were synthesized in moderate to excellent yield through a simple reaction of 2-aminoacetophenones with isocyanates or isothiocyanates. The reaction shows good tolerance of many important functional groups in the presence of air and water under metal-free conditions. Only water is produced as a coproduct, rendering this "green" methodology a highly versatile and eco-friendly alternative to the existing methods for the construction of the quinazolinone/quinazolinthione framework. We have interpreted the reaction mechanism by use of quantum chemical calculations on the basis of state-of-the-art computational methods SMD-B3LYP-D3(BJ)/BS1//B3LYP/BS1.

Keywords: cyclization, quinazolinone, quinazolinthione, quantum chemical calculations, SMD-B3LYP

\section{INTRODUCTION}

Quinazolinones and their derivatives occur frequently in natural and synthetic pharmaceutical products. Such compounds act as inhibitors of inducible and neuronal nitric oxide synthases (Camacho et al., 2016), possess anti-melanogenesis activity (Thanigaimalai et al., 2010), inhibit aldosterone synthase (CYP11B) and act as antagonists of platelet activating factor (Walser et al., 1991; Grombein et al., 2015). In recent years, many studies have been directed toward the design of 3,4-dihydroquinazolin-2(1H)-ones for the modulation of cardiotonic contractility (Campbell et al., 1988), inhibition of methionyl-tRNA synthetase (Buckner et al., 2015) and inhibition of HIV-1 Tat-TAR (Zeiger et al., 2014) together with the design of highly selective naked-eye sensors (Mei et al., 2012).

Many 3,4-dihydroquinazolin-2(1H)-one derivatives have been previously synthesized (Huang et al., 2008; Khan et al., 2014, 2015, 2016; Kshirsagar, 2015; Camacho et al., 2016; Maiden and Harrity, 2016; Awad et al., 2018; Zhang et al., 2018; Elkholy et al., 2019; Gatadi et al., 2019; Long et al., 2019; Wang et al., 2019). For example, Wang et al. (2009) reported the synthesis of 4-alkyl-2(1H)-quinazolinones via the cyclization of 1-(2-alkynyl-phenyl)ureas catalyzed by TfOH and Saunthwal et al. (2015) described a green and catalyst-free straightforward tandem synthesis of functionalized tetrahydroquinazolines from 2-aminophenylacrylate. Sawant et al. (2015) developed a microwave-promoted sequential cyclization-Mannich reaction of ketones, $o$-formyl carbamates 
and primary amines to form polyfunctionalized 3,4dihydroquinazolinones. Fukamachi et al. (2010) described a selective construction of 3,4-dihydroquinazoline-2-thiones by reacting 3-(2-isothiocyanatophenyl)propanoic derivatives with primary amines. 3,4-Dihydroquinazoline-2-thiones can also be synthesized from isothiocyanates with 2-aminophenyl acrylates or 2-amino chalcones (Hua et al., 2014; Xie et al., 2016). However, there are very few reports on the production of 4-alkenylquinazolinones and 4-alkenylquinazolinthiones. The first 4-alkenylquinazolinone to be successfully synthesized starting from quinoline-1-carboxamides was achieved in the presence of strong acids (Brack, 1969). Molina et al. (1993) utilized iminophosphorane and 2 equivalents of isocyanate to form 4-methylen-4H-3,1-benzoxazine which upon heating undergoes elimination and rearrangement to furnish 3-substituted 4-methenylquinazolinones. There were only two pioneering methodologies for the synthesis of 4-methylen-3,4-dihydroquinazolin-2-ones, one from 1-(oalkynylaryl)ureas catalyzed by an $\mathrm{Au}(\mathrm{I})$-complex (Scheme 1, top) (Gimeno et al., 2010, 2014a,b) and the other by reaction of 2-aminoacetophenone with the cyanomethyl anion electrogenerated by acetonitrile reduction at a graphite electrode (Scheme 1, middle) (Sbei et al., 2018). However, these reactions suffer from one or more drawbacks, such as expensive catalysts/ligands, harsh reaction conditions and multi-step preparation of raw materials. Thus, simple and efficient approaches to 4-alkenylquinazolinons and 4alkenylquinazolinthione synthesis from readily available starting materials remains an important target. Herein, we report the synthesis of 4-alkenylquinazolinones and 4alkenylquinazolinthiones from readily available materials (Scheme 1, bottom). The process is simple, proceeds under mild conditions in the absence of a catalyst for the synthesis of 4-alkenylquinazolinthiones and with catalytic amounts of $\mathrm{NaOH}$, for the synthesis of 4-alkenylquinazolinones.

\section{RESULTS AND DISCUSSION}

Nucleophilic addition of 2-aminoacetophenones $\mathbf{1}$ with isothiocyanates $\mathbf{2 a}$ results in a formation of 1,3-diarylthiourea 3. In principle, heterocyclization of compound $\mathbf{3}$ may occur by three different mechanisms (Scheme 2). Firstly, quinazolinthione 4a can result from the nucleophilic attack of N-3 of compound 3 on the carbonyl group of the ketone followed by removal of water (path A). Secondly, compound $\mathbf{3}$ is transformed to its mesomeric form $\mathbf{C}$ and the latter cyclizes through an addition reaction to the carbonyl group of the ketone leading to the formation of benzothiazinimine $4^{\prime}$ by the elimination of one molecule of water (path B). Thirdly, compound 3 is first converted to an enol form and the enol of intermediate $\mathbf{F}$ attacks the thiocarbonyl group of thiourea followed by a loss of $\mathrm{H}_{2} \mathrm{~S}$ to afford quinolinone $4^{\prime \prime}$ (path C).

In an initial experiment, the reaction of 2-aminoacetophenone $\mathbf{1}$ with phenyl isothiocyanate $\mathbf{2 a}$ was undertaken in acetonitrile $(\mathrm{MeCN})$ at room temperature. Not surprisingly, the main nucleophilic addition product 3 was isolated (yield: 50\%). Apart from this, a small proportion of a new compound was detected (entry 1, Table 1). ${ }^{1} \mathrm{H}$ NMR spectra demonstrated that there are two double peaks at 4.82 (one proton) and $3.69 \mathrm{ppm}$ (one proton), respectively, ruling out the existence of product $4^{\prime \prime}$. To identify the product structure as being either $\mathbf{4 a}$ or $\mathbf{4}^{\prime}$, single crystals were isolated and the structure characterized by X-ray crystallographic analysis. It was established that the quinazolinthione 4a was the correct structure (CCDC1906269, Figure 1, left, see Supporting Information for details). However, to our surprise, when crystals of the compound were incubated in methanol, an additional methoxy group was attached to 4position of the quinazolinthione ring (CCDC1906268, Figure 1, right), indicating that the methylene group of compound $\mathbf{4 a}$ is an active site and performs a further coupling reaction. This observation is currently under investigation.

Consistent with Fukui's theory, LUMO (the lowest unoccupied molecular orbital) and $\mathrm{E}_{\mathrm{LUMO}}$ (the energy of LUMO) are clear, as is the extent of molecular susceptibility toward attack by external electrons (Dhami et al., 1997; Yan et al., 2019). Thus, the carbon-carbon double bond character of the 4a structure is also represented by strong binding interactions in LUMO and LUMO+1 (Figure 2). This calculation provides a realistic description of the nucleophilic attack on the double bond on 4 a.

We optimized the reaction conditions in order to obtain the best yield by screening solvents and temperatures. As shown in Table 1, the yield was improved to $58 \%$ when the reaction was increased to $50^{\circ} \mathrm{C}$ (entry 2). In parallel, replacement of acetonitrile with ethyl acetate resulted in lower yield, even with an extended time. The reaction was found to occur with high efficiency at a short reaction time in acetonitrile under reflux conditions (entry 4). Replacement of acetonitrile with other solvents such as ethyl acetate, dichloromethane, THF, benzene and dioxane, resulted in lower yields (entries 5-9). Based on these results, the optimal reaction conditions were selected as $\mathbf{1}$ (1 $\mathrm{mmol})$ and $2(1.1 \mathrm{mmol})$ in $\mathrm{MeCN}(5 \mathrm{~mL})$ under reflux condition for $1.5 \mathrm{~h}$.

With these optimized reaction conditions in hand, the substrate scope of this methodology was explored (Figure 3). We found that both electron-donating groups (EDG) and electron-withdrawing groups (EWG) on the aromatic ring were well-tolerated by the reaction conditions. However, the yields of the products attached with EDG were significantly lower than those with EWG (4b-4d vs. $\mathbf{4 e - 4 g}$ ). In addition, the stereochemistry also influenced the yield. For example, using para- and meta-chloro substituted $\mathbf{2}$ in the reaction provided good yields of 4 whereas ortho-chloro substituted 2 gave the corresponding product in only a moderate yield (4e-g). The yield sequence followed the order: para- $>$ meta- $>$ ortho-. In similar fashion to mono-substituted analogs, di-substituted isothiocyanates reacted smoothly with 2 -aminoacetophenone to furnish the corresponding quinazolinthiones in moderate to high yields (4h-4i). Again, the electronic nature of the aryl moiety on the isothiocyanates had a dramatic effect on the product yield. As a result, 2,4-dichloro substituted $2 \mathbf{i}$ gave the corresponding product at $90 \%$ yield while the 2-methoxy-5-methyl substituted analog $\mathbf{2 h}$ only offered $42 \%$ yield of product $\mathbf{4 h}$. To further 


\section{previous work:}<smiles>[R]NC(=O)Nc1ccccc1C#C</smiles>

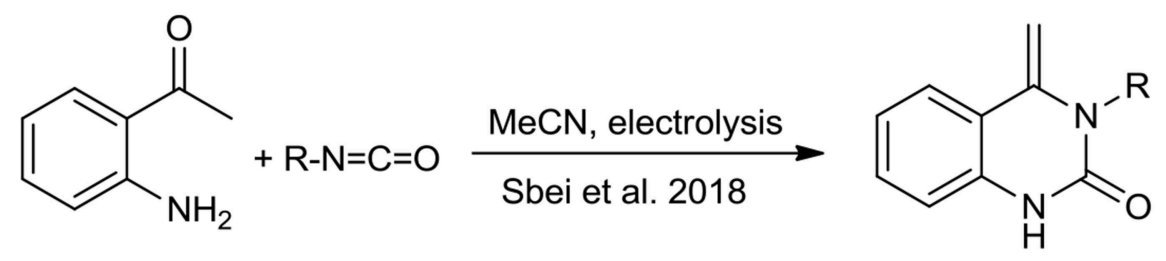

this work:

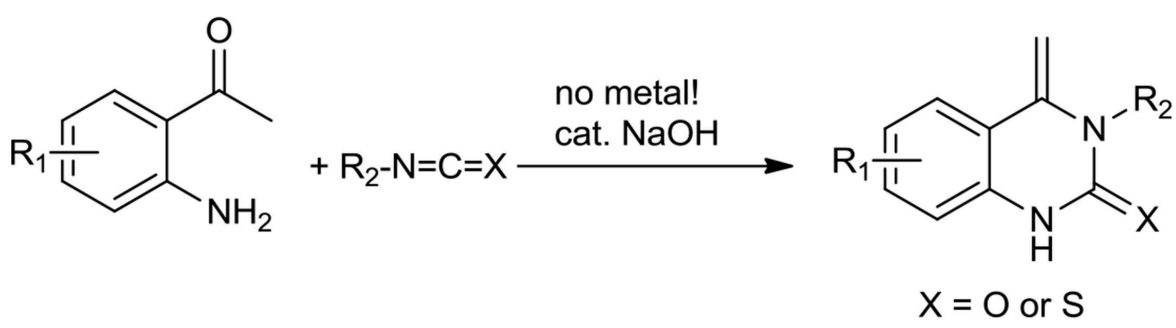

SCHEME 1 | Previous and present work for the synthesis of 4-methylene-quinazolin-2-ones.

expand the substrate scope, two aliphatic isothiocyanates $(\mathbf{2 j}$, 2k) were also investigated for this cycloaddition reaction. Benzyl isothiocyanate $(\mathbf{2} \mathbf{j})$ was found to be smoothly converted to the corresponding product $\mathbf{4} \mathbf{j}$ in a high yield, whereas propyl isothiocyanate (2k) only gave $20 \%$ yield of the desired $4 \mathbf{k}$. In addition, diisothiocyanate (2l) was also examined for this conversion, but only one isothiocyanate group was involved in the reaction and a mono-cyclized product $\mathbf{4 1}$ was obtained with the second isothiocyanate not involved in the reaction, even in the presence of an excess of 2 -aminoacetophenone 1 ( $>2$ equiv.). This interesting result provides an opportunity for further diversification and amplification with the entire isothiocyanate group. In this regard, compound $\mathbf{4 1}$ was refluxed with ethylamine, a highly nucleophilic amine, to afford compound $4 \mathbf{1}^{*}$ with an excellent yield (Scheme 3).

This success led us to further investigate the reaction generality by replacement of isothiocyanates with isocyanates. Under the optimized reaction conditions, the reaction of isocyanate $\mathbf{5}$ and 2-aminoacetophenone $\mathbf{1}$ only resulted in the coupling product $\mathbf{6}$, even after prolonging the reaction time to $24 \mathrm{~h}$. The annulation compound 7 was not detected (Scheme 4). Pleasingly, compound $\mathbf{6}$ can undergo an intramolecular cyclization to afford the target 4-methylene-quinazolinone 7 in the presence of $\mathrm{NaOH}(10 \mathrm{~mol} \%)$. Encouraged by these results, a subsequent study was undertaken to evaluate a one-pot cascade reaction of isocyanates 5 and 2-aminoacetophenone $\mathbf{1}$. In the presence of a catalytic amount of aqueous $\mathrm{NaOH}(10 \mathrm{~mol} \%)$, the reaction progressed smoothly to yield the annulation product 7 even at room temperature. Consequently we modified the conditions for the reaction of $\mathbf{1}$ with $\mathbf{2}$, and achieve a significantly improved yield for the production of $\mathbf{4 b}$ ( 58 vs. $10 \%$, Figure 3 ).

On the basis of this latter result, the scope of substituted isocyanates was explored (Figure 4). Aromatic isocyanates bearing either an EDG or an EWG group were found to undergo the reaction smoothly and the corresponding 4-methylenequinazolinones were formed in excellent yields $(\mathbf{7 b}-\mathbf{7} \mathbf{j})$. In this case, neither the electronic nature nor position of the functional groups attached to the aromatic ring dramatically influenced the product yield. However, the $2-\mathrm{CF}_{3}$-substituted isocyanate $5 \mathrm{k}$ only gave a moderate yield of the expected product $7 \mathbf{k}$. Compared to the mono-substituted analogs, di-substituted aryl isocyanates were converted into the corresponding quinolinones in relatively low yields (71-7o). In addition, three aliphatic isocyanates (5p5r) were investigated for this annulation reaction. It was found that benzyl isocyanate was converted smoothly to afford the corresponding product $\mathbf{7 p}$ in a high yield, whereas only moderate yields were achieved for $\mathbf{7 q}$ and $\mathbf{7 r}$. The diisocyanate (5s) was also investigated for this conversion. In a similar fashion to the 


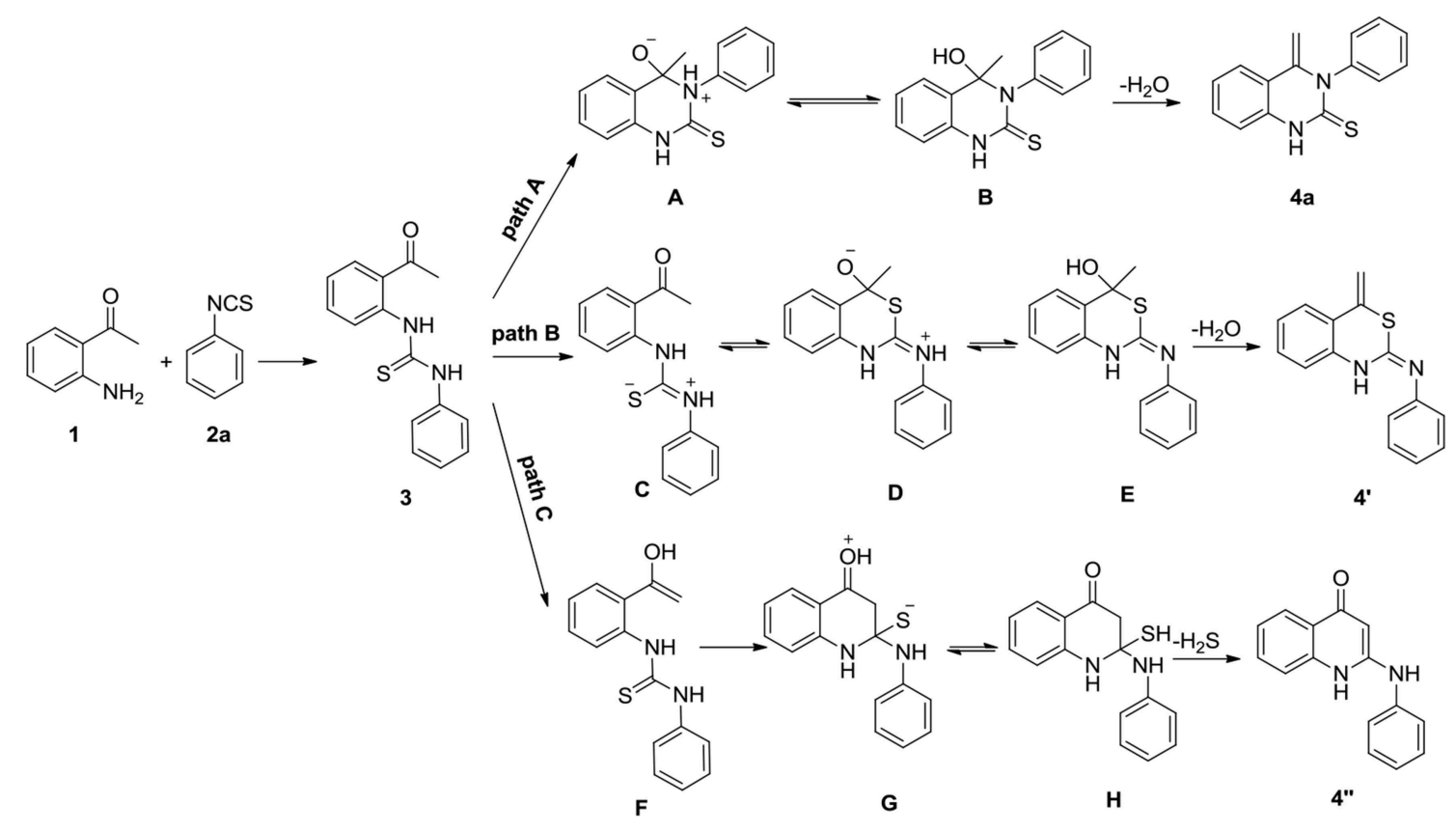

SCHEME 2 | Possible main pathways for the addition/cyclization of compounds $\mathbf{1}$ and $\mathbf{2 a}$.

TABLE 1 | Optimization of reaction conditions ${ }^{a}$.<smiles>CC(=O)c1ccccc1N</smiles>

1<smiles>O=[N+]C1=CC=[C+]C=C1</smiles>

$2 a$<smiles>C=C1c2ccccc2NC(=S)N1c1ccccc1</smiles>

$4 a$

\begin{tabular}{llcc}
\hline Entry & Solvent/T ( $\left.{ }^{\circ} \mathbf{C}\right)$ & Time (h) & Yield (\%) \\
\hline 1 & MeCN/20 & 20 & 10 \\
2 & MeCN/50 & 12 & 58 \\
3 & EtOAc/50 & 24 & 45 \\
$\mathbf{4}$ & MeCN/reflux & $\mathbf{1 . 5}$ & $\mathbf{9 6}$ \\
5 & EtOAc/reflux & 12 & 60 \\
6 & DCM/reflux & 8 & 25 \\
7 & THF/reflux & 5 & 39 \\
8 & Benzene/reflux & 5 & 64 \\
9 & Dioxane/reflux & 5 & 50 \\
\hline
\end{tabular}

${ }^{a}$ Reaction conditions: $1(1 \mathrm{mmol})$ and $\mathbf{2 a}(1.1 \mathrm{mmol})$ in a solvent $(5 \mathrm{~mL})$ at the indicated reaction conditions. Bold value indicates the best condition of the reaction.

aforementioned reaction with diisothiocyanate (21), only one isocyanate group was involved in the reaction and an excellent yield of mono-cyclized product $7 \mathrm{~s}$ was obtained.

The scope of substituted 2-aminoacetophenones $\mathbf{1}$ was also investigated in this novel cyclization process. 2Aminoacetophenones bearing either an EWG such as chloro group or an EDG such as methyl group on the aromatic ring, progressed in this reaction smoothly with either aromatic isocyanates or benzyl isocyanate to provide the corresponding quinazolinones in moderate to excellent yield (7t-7ab).

In order to demonstrate the suitability of this new synthetic methodology for industrial use, an increased scale preparation of $7 \mathbf{a}$ was investigated. Reaction of substrates $\mathbf{1}$ and $\mathbf{5 a}$ in $\mathrm{MeCN}$ $(20 \mathrm{mmol}, 50 \mathrm{~mL})$ was performed in the presence of $\mathrm{NaOH}(5 \mathrm{M}$ in $\mathrm{H}_{2} \mathrm{O}, 10 \mathrm{~mol} \%$ ) at room temperature. The corresponding product $7 \mathbf{a}$ was afforded in $90 \%$ yield, the yield being similar to that of the small-scale reaction (Scheme 5). Moreover, the workup procedure is simple, as the product precipitated from $\mathrm{MeCN}$ on cooling the reaction medium. A pure product was obtained after filtration, without the requirement of further purification.

As indicated in Scheme 2, the heterocyclization of starting materials 1 and 2/5 may in principle lead to at least three different products. To better understand the intramolecular cyclization reaction, we calculated Gibbs free energy of each intermediate and products listed in Scheme 2 by a computational method. Compounds $\mathbf{1}$ and $\mathbf{2 a}$ were selected as the model substrates for this study. The calculation was based on quantum chemical calculations (SMD-B3LYP-D3(BJ)/BS1//B3LYP/BS1). The geometry of transition states (TSs) and compound 3 in Scheme 2 have been fully optimized in vacuum and the TSs were verified to have only one imaginary frequency vibrational mode that connects the reactants and products. The calculated energy values of the intermediates and products, including dispersion interactions are presented in Figure 5. All stationary points were fully optimized and subjected to frequency analyses. The data demonstrates that paths $\mathrm{A}$ and $\mathrm{C}$ mechanism routes have a negative value of Gibbs free energy for the final product while the path $\mathrm{B}$ mechanism route gives a positive value, thus ruling 


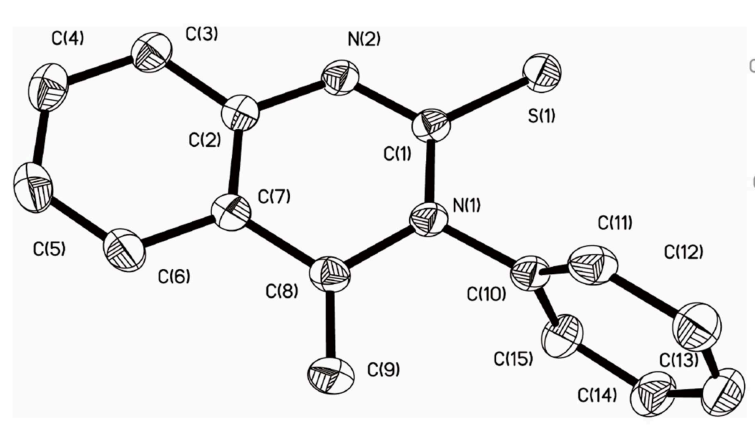

$4 a$

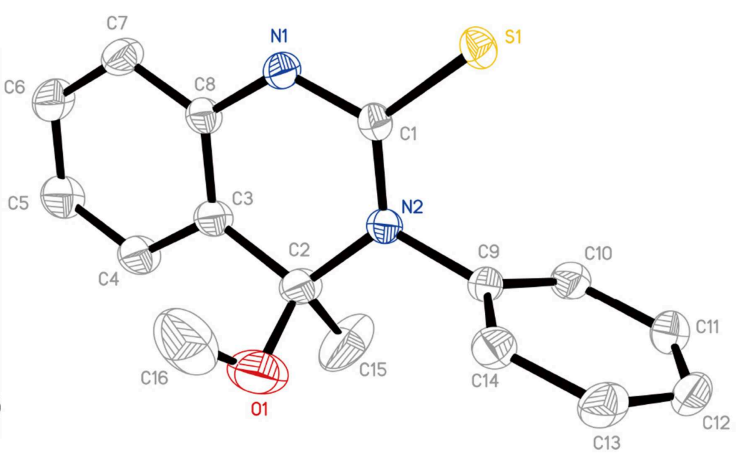

$4 a^{*}$

FIGURE 1 | Single crystal X-ray structures of compound $\mathbf{4 a}$ and its derivative $\mathbf{4 a *}$.
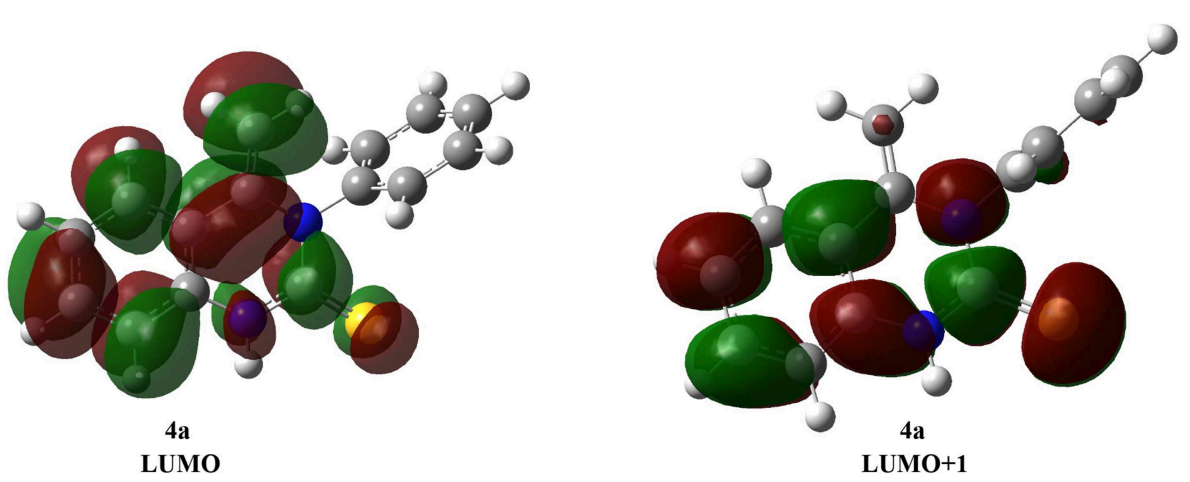

FIGURE 2 | The optimized Structure of $\mathbf{4 a}$ LUMO and LUMO+1 orbital.

out the path $\mathrm{B}$ mechanism. In the route of path $\mathrm{A} 3 \rightarrow$ $\mathrm{A} \rightarrow \mathrm{B} \rightarrow$ 4a (black, Figure 5), there is only one energy barrier at a value of $4.77 \mathrm{kcal} / \mathrm{mol}$. In contrast, there are two high energy barriers $(20.53$ and $7.19 \mathrm{kcal} / \mathrm{mol})$ in the route of path $\mathrm{C} \mathbf{3} \rightarrow \mathrm{F} \rightarrow \mathrm{G} \rightarrow \mathrm{H} \rightarrow \mathbf{4}^{\prime \prime}$ (blue), rendering the transformation via path $\mathrm{C}$ difficult. These results indicate that the route of path $\mathrm{A}$ is the most probable pathway for this annulation reaction.

\section{CONCLUSION}

In summary, we have presented a novel preparation of 3-substituted 4-methylene-3,4-dihydroquinazoline2(1H)-thiones/ones from 2-aminoacetophenone and isothiocyanates/isocyanates. The method utilizes commercially available starting materials and is applicable to a large range of compounds. This new synthetic methodology does not require expensive/complicated metal catalysts and the work-up procedure is simple. The novel transformation indicates the feasibility of this pathway in both research and industrial laboratories. Comprehensive quantum chemical calculation studies indicate the probable synthetic route.

\section{MATERIALS AND METHODS}

\section{Experimental and Computational Details}

All chemicals were obtained from Aladdin (China) as reagent grade and were used as received. Column chromatography purifications were performed on silica gel $60(0.04-0.063 \mathrm{~mm})$. Melting points were determined using an Electrothermal WRS1B Digital Melting Point Apparatus and are uncorrected. ${ }^{1} \mathrm{H}$ NMR spectra were recorded using a Bruker $(400 / 500 \mathrm{MHz})$ NMR spectrometer. ${ }^{13} \mathrm{C}-\mathrm{NMR}$ spectra were recorded using a Bruker (101/151 MHz) NMR spectrometer. Chemical shifts ( $\delta$ ) are reported in ppm downfield from the internal standard tetramethylsilane (TMS).

Gaussian 09 software (Frisch et al., 2016) packages can implement density functional theory (DFT) conveniently, thus geometry optimizations were carried out with the hybrid B3LYP functional in conjunction with $6-31 \mathrm{G}++(\mathrm{d}, \mathrm{p})$ basis set for all the atoms of these compounds. Additionally, the geometry optimizations were followed by frequency calculations using the same basis set. Moreover, the effect of dispersion was incorporated using Grimme-D3 approximation during geometry optimizations (Grimme et al., 2010). To further refine the energies, single-point B3LYP calculations including D3 version of Grimme's dispersion with Becke-Johnson damping (D3BJ) 


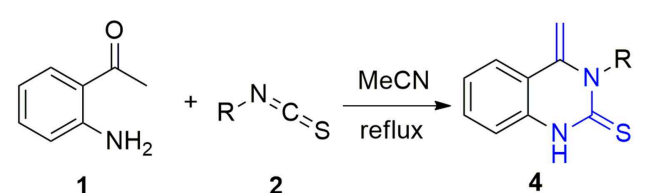

1<smiles>C=C1c2ccccc2NC(=S)N1c1ccccc1</smiles><smiles>C=C1c2ccccc2NC(=S)N1c1ccccc1C</smiles><smiles>C=C1c2ccccc2NC(=S)N1c1cccc(OC)c1</smiles><smiles>C=C1c2ccccc2NC(=S)N1c1ccc(OC)cc1</smiles>

4a (98\%)

4b $\left(10 \%, 58 \%^{c}\right)$

4c $(41 \%)$

$$
\text { 4d (53\%) }
$$<smiles>C=C1c2ccccc2NC(=S)N1c1ccccc1Cl</smiles>

4e $(65 \%)$<smiles>C=C1c2ccccc2NC(=S)N1c1cccc(Cl)c1</smiles>

4f $(75 \%)$<smiles>C=C1c2ccccc2NC(=S)N1c1ccc(Cl)cc1Cl</smiles>

4i $(90 \%)$<smiles>C=C1c2ccccc2NC(=S)N1Cc1ccccc1</smiles>

4j (90\%)<smiles>C=C1c2ccccc2NC(=S)N1c1ccc(C)cc1</smiles>

4 g $(78 \%)$<smiles>C=C1c2ccccc2NC(=S)N1CCC</smiles>

4k (20\%)<smiles>C=C1c2ccccc2NC(=S)N1c1cc(C)ccc1OCC(C)(C)C</smiles>

4h $(42 \%)$<smiles>C=C1c2ccccc2NC(=S)N1c1ccc(N(C)SC)cc1</smiles>

4I $(76 \%)$

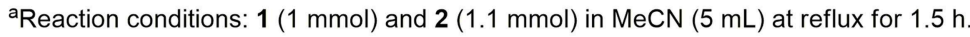<smiles></smiles>

41

\section{Ethylamine DCM, reflux}<smiles>C=C1c2ccccc2NC(=S)N1c1ccc(NC(=S)NCC)cc1</smiles>

$4 I^{*}(98 \%)$

SCHEME 3 | Nucleophilic attack of $\mathbf{4 l}^{\star}$ by an aliphatic amine.

(Grimme et al., 2011) were performed with a higher basis set BS1 (BS1 $=6-31++\mathrm{G}(\mathrm{d}, \mathrm{p})$ basis set for all atoms). The use of SMDB3LYP-D3(BJ)/BS1 to calculate compound Gibbs energy was reported in the literature for mechanistic investigation (Markovic et al., 2010; Kleine et al., 2011).

\section{Chemical Synthesis}

\section{Synthesis of $4 a-41$}

A mixture of 2-aminoacetophenone $\mathbf{1}\left(\begin{array}{lll}1 & \mathrm{mmol})\end{array}\right)$ and isothiocyanatobenzene $2(1.1 \mathrm{mmol})$ in acetonitrile $(5 \mathrm{ml})$ was stirred at $82^{\circ} \mathrm{C}$ for $4 \mathrm{~h}$. After completion, the reaction solvent was reduced to half and upon cooling a white or yellow precipitate was obtained. Recrystallization from ethanol produced white or yellow solid.

\section{4-Methylene-3-phenyl-3,4-dihydroquinazoline-2(1H)-} thione (4a)

Yellow solid, m.p. $217 \sim 218^{\circ} \mathrm{C} ;{ }^{1} \mathrm{H}$ NMR $(400 \mathrm{MHz}$, Chloroformd) $\delta 9.98(\mathrm{~s}, 1 \mathrm{H}), 7.56(\mathrm{dd}, J=8.3,6.8 \mathrm{~Hz}, 2 \mathrm{H}), 7.52-7.45(\mathrm{~m}$, $2 \mathrm{H}), 7.31-7.26(\mathrm{~m}, 3 \mathrm{H}), 7.24(\mathrm{~d}, J=1.3 \mathrm{~Hz}, 1 \mathrm{H}), 7.07(\mathrm{td}, J=7.7$, $1.2 \mathrm{~Hz}, 1 \mathrm{H}), 6.91(\mathrm{dd}, J=8.0,1.1 \mathrm{~Hz}, 1 \mathrm{H}), 4.82(\mathrm{~d}, J=2.6 \mathrm{~Hz}$, $1 \mathrm{H}), 3.69(\mathrm{~d}, J=2.6 \mathrm{~Hz}, 1 \mathrm{H}) .{ }^{13} \mathrm{C}$ NMR $(101 \mathrm{MHz}$, Chloroformd) $\delta 174.18,141.83,141.66,133.23,130.65,130.07,128.94,128.67$, 


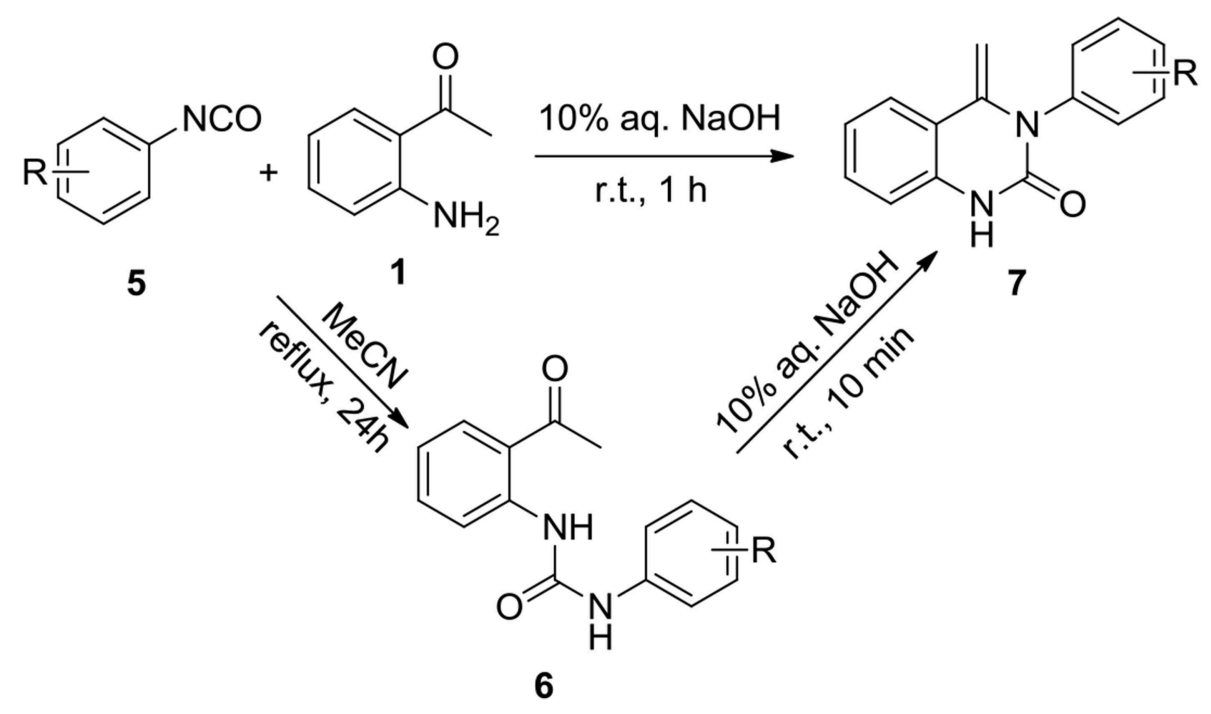

SCHEME 4 | Coupling and annulation reaction of $\mathbf{5}$ and $\mathbf{6}$.

124.40, 123.83, 118.26, 114.84, 90.14. HRMS (ESI): Calc. for $\mathrm{C}_{15} \mathrm{H}_{12} \mathrm{~N}_{2} \mathrm{~S}[\mathrm{M}+\mathrm{H}]^{+}:$253.0794, found 253.2068.

\section{4-Methylene-3-(o-tolyl)-3,4-dihydroquinazoline-2(1H)- thione (4b)}

Brown powder, m.p. $214 \sim 215^{\circ} \mathrm{C}$; ${ }^{1} \mathrm{H}$ NMR (500 MHz, DMSO$\left.d_{6}\right) \delta 11.64(\mathrm{~s}, 1 \mathrm{H}), 7.68(\mathrm{~d}, J=8.4 \mathrm{~Hz}, 1 \mathrm{H}), 7.40-7.36$ $(\mathrm{m}, 2 \mathrm{H}), 7.36-7.32(\mathrm{~m}, 2 \mathrm{H}), 7.16-7.11(\mathrm{~m}, 2 \mathrm{H}), 7.09(\mathrm{dd}, J$ $=7.9,1.2 \mathrm{~Hz}, 1 \mathrm{H}), 4.89(\mathrm{~d}, J=2.1 \mathrm{~Hz}, 1 \mathrm{H}), 3.38(\mathrm{~d}, J=$ $2.2 \mathrm{~Hz}, 1 \mathrm{H}), 2.13(\mathrm{~s}, 3 \mathrm{H}) .{ }^{13} \mathrm{C}$ NMR $\left(126 \mathrm{MHz}, \mathrm{DMSO}-d_{6}\right) \delta$ $173.38,140.67,140.62,135.83,134.15,131.68,131.29,129.36$, $128.86,128.10,124.49,124.42,117.65,115.58,88.04,17.16$. HRMS (ESI) $m / z$ : Calc. for $\mathrm{C}_{16} \mathrm{H}_{14} \mathrm{~N}_{2} \mathrm{~S}[\mathrm{M}+\mathrm{H}]^{+}:$267.0950, found 257.0933 .

\section{3-(3-Methoxyphenyl)-4-methylene-3,4-dihydroquinazoline- 2(1H)-thione (4c)}

Yellow powder, m.p. 221 225 ${ }^{\circ} \mathrm{C}$; ${ }^{1} \mathrm{H}$ NMR (500 MHz, DMSO$\left.d_{6}\right) \delta 11.63(\mathrm{~s}, 1 \mathrm{H}), 7.66(\mathrm{dd}, J=8.1,1.3 \mathrm{~Hz}, 1 \mathrm{H}), 7.47-7.41$ $(\mathrm{m}, 1 \mathrm{H}), 7.37(\mathrm{ddd}, J=8.4,7.3,1.3 \mathrm{~Hz}, 1 \mathrm{H}), 7.14(\mathrm{dd}, J=$ 8.1, $1.2 \mathrm{~Hz}, 1 \mathrm{H}$ ), 7.09 (ddd, $J=8.3,7.3,1.2 \mathrm{~Hz}, 1 \mathrm{H}$ ), 7.03-6.97 $(\mathrm{m}, 1 \mathrm{H}), 6.83-6.79(\mathrm{~m}, 2 \mathrm{H}), 4.92(\mathrm{~d}, J=2.2 \mathrm{~Hz}, 1 \mathrm{H}), 3.77(\mathrm{~s}$, $3 \mathrm{H}), 3.51(\mathrm{~d}, J=2.2 \mathrm{~Hz}, 1 \mathrm{H}) .{ }^{13} \mathrm{C}$ NMR $\left(126 \mathrm{MHz}, \mathrm{DMSO}-d_{6}\right)$ $\delta 173.95,160.93,143.12,141.84,134.13,131.20,130.93,124.40$, $124.36,121.71,118.04,115.51,115.30,114.15,89.25,55.81$. HRMS (ESI) $m / z$ : Calc. for $\mathrm{C}_{16} \mathrm{H}_{14} \mathrm{~N}_{2} \mathrm{OS}[\mathrm{M}+\mathrm{H}]^{+}$: 283.0900, found 283.0864 .

\section{3-(4-Methoxyphenyl)-4-methylene-3,4-dihydroquinazoline- 2(1H)-thione (4d)}

White powder, m.p. $212 \sim 214^{\circ} \mathrm{C} ;{ }^{1} \mathrm{H}$ NMR $(400 \mathrm{MHz}$, Chloroform-d) $\delta 9.19(\mathrm{~s}, 1 \mathrm{H}), 7.54(\mathrm{~d}, J=8.0 \mathrm{~Hz}, 1 \mathrm{H})$, $7.33(\mathrm{t}, J=7.9 \mathrm{~Hz}, 1 \mathrm{H}), 7.22(\mathrm{~d}, J=8.5 \mathrm{~Hz}, 2 \mathrm{H}), 7.16-7.04(\mathrm{~m}$, $3 \mathrm{H}), 6.87(\mathrm{~s}, 1 \mathrm{H}), 4.85(\mathrm{~d}, J=2.1 \mathrm{~Hz}, 1 \mathrm{H}), 3.89(\mathrm{~s}, 3 \mathrm{H}), 3.80$ $(\mathrm{d}, J=2.1 \mathrm{~Hz}, 1 \mathrm{H}) .{ }^{13} \mathrm{C}$ NMR $\left(126 \mathrm{MHz}, \mathrm{DMSO}-d_{6}\right) \delta 174.47$,
$159.01,142.36,134.93,134.14,131.18,130.57,124.44,124.34$, $118.05,115.50,115.39,89.18,55.76$. HRMS (ESI) $m / z$ : calc. for $\mathrm{C}_{16} \mathrm{H}_{14} \mathrm{~N}_{2} \mathrm{OS}[\mathrm{M}+\mathrm{H}]^{+}: 283.0900$, found 283.0912.

\section{3-(2-Chlorophenyl)-4-methylene-3,4-dihydroquinazoline- 2(1H)-thione (4e)}

Yellow powder, m.p. $203 \sim 205^{\circ} \mathrm{C}$; ${ }^{1} \mathrm{H}$ NMR (500 MHz, DMSO$\left.d_{6}\right) \delta 11.76(\mathrm{~s}, 1 \mathrm{H}), 7.70(\mathrm{~d}, J=8.0 \mathrm{~Hz}, 1 \mathrm{H}), 7.65(\mathrm{dd}, J=7.8$, $1.8 \mathrm{~Hz}, 1 \mathrm{H}), 7.55-7.45(\mathrm{~m}, 2 \mathrm{H}), 7.44-7.36(\mathrm{~m}, 2 \mathrm{H}), 7.17-7.08(\mathrm{~m}$, $2 \mathrm{H}), 4.93(\mathrm{~d}, J=2.6 \mathrm{~Hz}, 1 \mathrm{H}), 3.38(\mathrm{~d}, J=2.6 \mathrm{~Hz}, 1 \mathrm{H}) .{ }^{13} \mathrm{C} \mathrm{NMR}$ $\left(126 \mathrm{MHz}, \mathrm{DMSO}-d_{6}\right) \delta 173.56,140.37,138.85,133.99,132.29$, $132.00,131.38,130.96,130.54,129.24,124.56,124.52,117.66$, $115.68,88.17$. HRMS (ESI) $m / z$ : calc. for $\mathrm{C}_{15} \mathrm{H}_{11} \mathrm{ClN}_{2} \mathrm{~S}[\mathrm{M}+$ $\mathrm{H}]^{+}: 287.0404$, found 287.0389 .

\section{3-(3-Chlorophenyl)-4-methylene-3,4-dihydroquinazoline- 2(1H)-thione (4f)}

White powder, m.p. $234 \sim 236^{\circ} \mathrm{C} ;{ }^{1} \mathrm{H}$ NMR (400 MHz, DMSO$\left.d_{6}\right) \delta 11.79(\mathrm{~d}, J=2.5 \mathrm{~Hz}, 1 \mathrm{H}), 7.69(\mathrm{t}, J=7.7 \mathrm{~Hz}, 1 \mathrm{H}), 7.59$ (, $J=7.9,2.6 \mathrm{~Hz}, 1 \mathrm{H}), 7.56-7.49(\mathrm{~m}, 1 \mathrm{H}), 7.46-7.35(\mathrm{~m}, 2 \mathrm{H}), 7.27$ $(\mathrm{dt}, J=7.8,1.6 \mathrm{~Hz}, 1 \mathrm{H}), 7.21-7.16(\mathrm{~m}, 1 \mathrm{H}), 7.12(\mathrm{dd}, J=9.8$, $5.6 \mathrm{~Hz}, 1 \mathrm{H}), 4.96(\mathrm{~d}, J=4.1,2.2 \mathrm{~Hz}, 1 \mathrm{H}), 3.46(\mathrm{~d}, J=2.5 \mathrm{~Hz}, 1 \mathrm{H})$. ${ }^{13} \mathrm{C}$ NMR $\left(101 \mathrm{MHz}, \mathrm{DMSO}-d_{6}\right) \delta 173.93,143.32,141.91,134.21$, $134.06,131.86,131.30,129.90,128.81,128.77,124.49,124.42$, $118.03,115.64,89.35$. HRMS (ESI) $m / z$ : calc. for $\mathrm{C}_{15} \mathrm{H}_{11} \mathrm{ClN}_{2} \mathrm{~S}$ $[\mathrm{M}+\mathrm{H}]^{+}: 287.0404$, found 287.0423 .

\section{3-(4-Chlorophenyl)-4-methylene-3,4-dihydroquinazoline- 2(1H)-thione (4g)}

White powder, m.p. $240 \sim 245^{\circ} \mathrm{C}$; ${ }^{1} \mathrm{H}$ NMR (500 MHz, DMSO$\left.d_{6}\right) \delta 11.72(\mathrm{~s}, 1 \mathrm{H}), 7.68(\mathrm{dd}, J=8.2,1.4 \mathrm{~Hz}, 1 \mathrm{H}), 7.61-7.57(\mathrm{~m}$, 2H), 7.38 (ddd, $J=8.4,7.2,1.3 \mathrm{~Hz}, 1 \mathrm{H}), 7.31-7.26(\mathrm{~m}, 2 \mathrm{H}), 7.15$ $(\mathrm{dd}, J=8.1,1.2 \mathrm{~Hz}, 1 \mathrm{H}), 7.10(\mathrm{ddd}, J=8.3,7.3,1.3 \mathrm{~Hz}, 1 \mathrm{H})$, $4.94(\mathrm{~d}, J=2.4 \mathrm{~Hz}, 1 \mathrm{H}), 3.45(\mathrm{~d}, J=2.5 \mathrm{~Hz}, 1 \mathrm{H}) .{ }^{13} \mathrm{C} \mathrm{NMR}$ $\left(126 \mathrm{MHz}, \mathrm{DMSO}-d_{6}\right) \delta 174.01,141.96,141.00,134.06,133.08$, 
<smiles>[R17]c1ccc(N)c(C(C)=O)c1</smiles><smiles>C=C1c2ccccc2NC(=O)N1C(=C)c1ccccc1N1C(=C)c2ccccc2NC1=O</smiles><smiles>C=C1c2ccccc2NC(=O)N1c1ccccc1F</smiles>

7 e $(91 \%)$

$7 f(88 \%)$

$7 g(82 \%)$

7h $(82 \%)$<smiles>C=C1c2ccccc2NC(=O)N1c1cccc(Cl)c1</smiles><smiles>C=C1c2ccccc2NC(=O)N1c1c(CC)cccc1CC</smiles><smiles>C=C1c2ccccc2NC(=O)N1CCC</smiles><smiles>C=C1c2ccccc2NC(=O)N1C1CCCCC1</smiles><smiles>C=C1c2ccccc2NC(=O)N1c1ccc([N+](=O)[O-])cc1</smiles><smiles>C=C1c2cc(Cl)ccc2NC(=O)N1c1ccccc1F</smiles><smiles>C=C1c2cc(Cl)ccc2NC(=O)N1c1cccc(Cl)c1</smiles><smiles>C=C1c2cc(Cl)ccc2NC(=O)N1c1cccc(C)c1</smiles><smiles>C=C1c2cc(Cl)ccc2NC(=O)N1c1ccccc1C</smiles><smiles></smiles><smiles>C=C1c2ccc(C)cc2NC(=O)N1c1ccccc1F</smiles><smiles>[Z16][R15]([H])([H])[H]</smiles><smiles>C=C1c2ccc(C)cc2NC(=O)N1Cc1ccccc1</smiles><smiles>C=C1c2cc(Cl)ccc2NC(=O)N1Cc1ccccc1</smiles>

7 aa (52\%)

$7 a b$ (92\%)

aReaction conditions: 1 (1 mmol), $5(1.1 \mathrm{mmol})$ and $\mathrm{NaOH}\left(5 \mathrm{M}\right.$ in $\left.\mathrm{H}_{2} \mathrm{O}, 10 \mathrm{~mol} \%\right)$ in $\mathrm{MeCN}(5 \mathrm{~mL})$ at $20^{\circ} \mathrm{C}$ for $1 \mathrm{~h}$.

${ }^{\mathrm{b}}$ Isolated yield.

FIGURE 4 | Substrate scope of isocyanates ${ }^{a, b}$. 


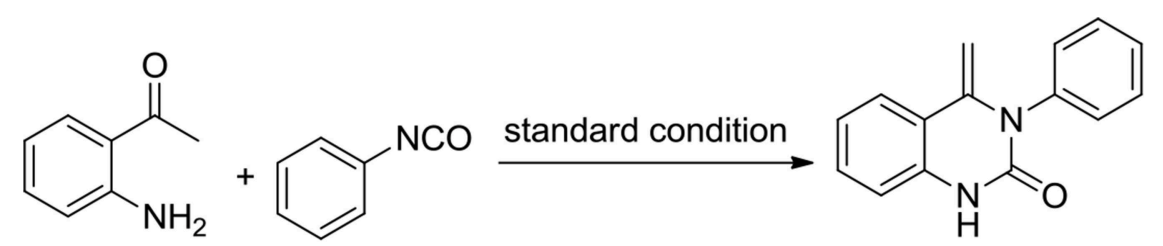

1

$5 a$

7a, $20 \mathrm{mmol}$ scale, $90 \%$

SCHEME 5 | Gram-scale preparation of $\mathbf{7 a}$.

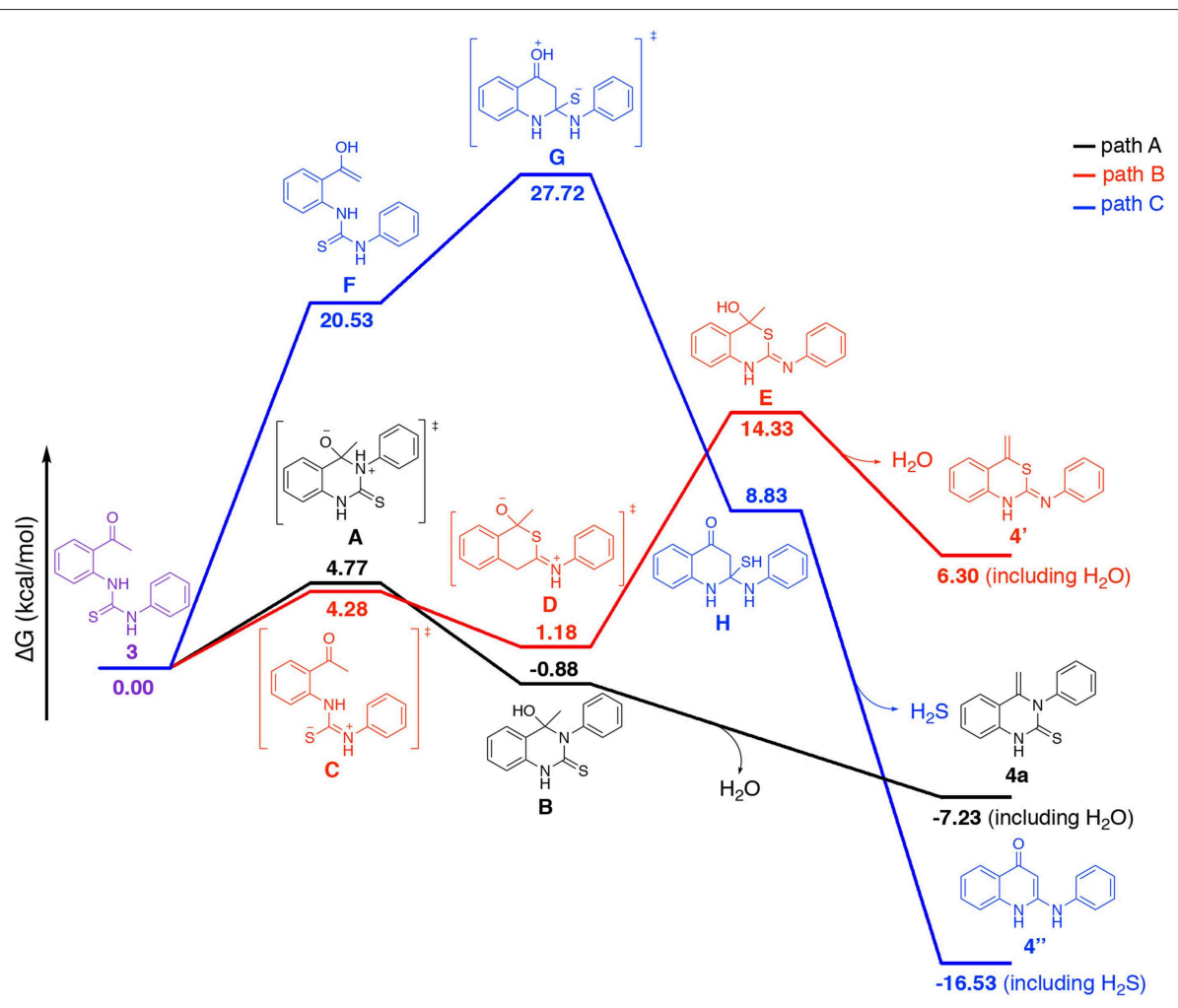

FIGURE 5 | Calculated comparative Gibbs free energy profile for three different mechanism routes. Red, Blue, and Black lines stand for the route of TS and optimized structures Gibbs energy change. Calculated Gibbs free energies relative to $\mathbf{3}$ at SMD-B3LYP-D3(BJ)/BS1//B3LYP/BS1 [kcal/mol] (see Supporting Information for optimized structures).

$131.74,131.30,130.43,124.49,124.45,118.00,115.61,89.33$. HRMS (ESI) $m / z$ : calc. for $\mathrm{C}_{15} \mathrm{H}_{11} \mathrm{ClN}_{2} \mathrm{~S}[\mathrm{M}+\mathrm{H}]^{+}: 287.0404$, found 287.0367 .

\section{3-(2-Methoxy-6-methylphenyl)-4-methylene-3,4- dihydroquinazoline-2(1H)-thione (4h)}

Yellow powder, m.p. 236 237 ${ }^{\circ} \mathrm{C}$; ${ }^{1} \mathrm{H}$ NMR (500 MHz, DMSO$\left.d_{6}\right) \delta 11.54(\mathrm{~s}, 1 \mathrm{H}), 7.64(\mathrm{dd}, J=8.1,1.3 \mathrm{~Hz}, 1 \mathrm{H}), 7.35$ (ddd, $J=8.4,7.3,1.3 \mathrm{~Hz}, 1 \mathrm{H}), 7.23-7.19(\mathrm{~m}, 1 \mathrm{H}), 7.12(\mathrm{dd}, J=$ 8.2, $1.2 \mathrm{~Hz}, 1 \mathrm{H}), 7.10-7.05(\mathrm{~m}, 2 \mathrm{H}), 6.95(\mathrm{dd}, J=2.2,0.8 \mathrm{~Hz}$, $1 \mathrm{H}), 4.82(\mathrm{~d}, J=2.1 \mathrm{~Hz}, 1 \mathrm{H}), 3.71(\mathrm{~s}, 3 \mathrm{H}), 3.50(\mathrm{~d}, J=$ $2.1 \mathrm{~Hz}, 1 \mathrm{H}), 2.28(\mathrm{~s}, 3 \mathrm{H}) .{ }^{13} \mathrm{C}$ NMR $\left(126 \mathrm{MHz}, \mathrm{DMSO}-d_{6}\right) \delta$ $174.12,152.82,140.82,134.17,131.13,130.83,130.60,130.52$, $129.80,124.42,124.30,117.86,115.47,113.43,87.98,56.21,20.42$.
HRMS (ESI) $m / z$ : calc. for $\mathrm{C}_{17} \mathrm{H}_{16} \mathrm{~N}_{2} \mathrm{OS}[\mathrm{M}+\mathrm{H}]^{+}:$297.1056, found 297.1023 .

\section{3-(2,4-Dichlorophenyl)-4-methylene-3,4-} dihydroquinazoline-2(1H)-thione (4i)

White powder, m.p. $236 \sim 237^{\circ} \mathrm{C} ;{ }^{1} \mathrm{H}$ NMR $(500 \mathrm{MHz}, \mathrm{DMSO}-$ $\left.d_{6}\right) \delta 11.82(\mathrm{~s}, 1 \mathrm{H}), 7.85(\mathrm{~d}, J=2.3 \mathrm{~Hz}, 1 \mathrm{H}), 7.71(\mathrm{dd}, J=8.1$, $1.3 \mathrm{~Hz}, 1 \mathrm{H}), 7.60(\mathrm{dd}, J=8.5,2.4 \mathrm{~Hz}, 1 \mathrm{H}), 7.48(\mathrm{~d}, J=8.5 \mathrm{~Hz}$, $1 \mathrm{H}), 7.39$ (ddd, $J=8.4,7.2,1.3 \mathrm{~Hz}, 1 \mathrm{H}), 7.18-7.13(\mathrm{~m}, 1 \mathrm{H}), 7.11$ $(\mathrm{dd}, J=8.1,1.1 \mathrm{~Hz}, 1 \mathrm{H}), 4.96(\mathrm{~d}, J=2.7 \mathrm{~Hz}, 1 \mathrm{H}), 3.45(\mathrm{~d}, J=$ $2.8 \mathrm{~Hz}, 1 \mathrm{H}) .{ }^{13} \mathrm{C}$ NMR $\left(126 \mathrm{MHz}, \mathrm{DMSO}-d_{6}\right) \delta 173.48,140.17$, $138.00,134.16,133.91,133.65,133.46,131.44,130.64,129.54$, $124.66,124.55,117.61,115.75,88.33$. HRMS (ESI) $m / z$ : calc. for $\mathrm{C}_{15} \mathrm{H}_{10} \mathrm{Cl}_{2} \mathrm{~N}_{2} \mathrm{~S}[\mathrm{M}+\mathrm{H}]^{+}: 321.0015$, found 321.0023 . 


\section{3-Benzyl-4-methylene-3,4-dihydroquinazoline-2(1H)- thione (4j)}

White powder, m.p. $241 \sim 242^{\circ} \mathrm{C}$ (Morgenstern and Richter, 1992, $215 \sim 217^{\circ} \mathrm{C}$ ); ${ }^{1} \mathrm{H}$ NMR $\left(500 \mathrm{MHz}, \mathrm{DMSO}-d_{6}\right) \delta 11.65(\mathrm{~s}, 1 \mathrm{H})$, $7.60(\mathrm{dd}, J=8.1,1.3 \mathrm{~Hz}, 1 \mathrm{H}), 7.37-7.27(\mathrm{~m}, 5 \mathrm{H}), 7.27-7.21(\mathrm{~m}$, $1 \mathrm{H}), 7.14(\mathrm{dd}, J=8.1,1.2 \mathrm{~Hz}, 1 \mathrm{H}), 7.07(\mathrm{ddd}, J=8.3,7.3,1.2 \mathrm{~Hz}$, $1 \mathrm{H}), 5.68(\mathrm{~s}, 2 \mathrm{H}), 4.98(\mathrm{~d}, J=2.9 \mathrm{~Hz}, 1 \mathrm{H}), 4.31(\mathrm{~d}, J=2.9 \mathrm{~Hz}$, $1 \mathrm{H}) .{ }^{13} \mathrm{C}$ NMR $\left(126 \mathrm{MHz}, \mathrm{DMSO}-d_{6}\right) \delta 174.87,138.11,136.39$, $133.73,131.01,128.93,127.28,126.59,124.59,124.27,118.02$, $115.42,89.39,52.81$. HRMS (ESI) $m / z$ : calc. for $\mathrm{C}_{16} \mathrm{H}_{14} \mathrm{~N}_{2} \mathrm{~S}[\mathrm{M}$ $+\mathrm{H}]^{+}: 267.0950$, found 267.0932.

\section{4-Methylene-3-propyl-3,4-dihydroquinazoline-2(1H)- thione (4k)}

White powder, m.p. $168 \sim 169^{\circ} \mathrm{C} ;{ }^{1} \mathrm{H}$ NMR (500 MHz, DMSO- $\left.d_{6}\right)$ $\delta 11.35(\mathrm{~s}, 1 \mathrm{H}), 7.65(\mathrm{dd}, J=8.3,1.3 \mathrm{~Hz}, 1 \mathrm{H}), 7.33-7.28(\mathrm{~m}, 1 \mathrm{H})$, $7.08-7.04(\mathrm{~m}, 2 \mathrm{H}), 5.05(\mathrm{~d}, J=2.9 \mathrm{~Hz}, 1 \mathrm{H}), 4.52(\mathrm{~d}, J=2.9 \mathrm{~Hz}$, $1 \mathrm{H}), 4.25$ (s, 2H), $1.78-1.68(\mathrm{~m}, 2 \mathrm{H}), 0.92(\mathrm{t}, J=7.4 \mathrm{~Hz}, 3 \mathrm{H})$. ${ }^{13} \mathrm{C}$ NMR $\left(126 \mathrm{MHz}, \mathrm{DMSO}-d_{6}\right) \delta 173.70,138.33,133.85,130.88$, 124.37, 124.34, 118.06, 115.18, 87.82, 50.78, 18.16, 11.31. HRMS (ESI) $m / z:\left(\mathrm{M}+\mathrm{H}^{+}\right)$; calc. for $\mathrm{C}_{12} \mathrm{H}_{14} \mathrm{~N}_{2} \mathrm{~S}[\mathrm{M}+\mathrm{H}]^{+}: 219.0950$, found 219.0938 .

\section{3-(4-Isothiocyanatophenyl)-4-methylene-3,4- dihydroquinazoline-2(1H)-thione (4l)}

Yellow powder, m.p. $236 \sim 239^{\circ} \mathrm{C}$; ${ }^{1} \mathrm{H}$ NMR (500 MHz, DMSO$\left.d_{6}\right) \delta 11.74(\mathrm{~s}, 1 \mathrm{H}), 7.68(\mathrm{dd}, J=8.1,1.3 \mathrm{~Hz}, 1 \mathrm{H}), 7.61-7.56$ $(\mathrm{m}, 2 \mathrm{H}), 7.38(\mathrm{ddd}, J=9.5,6.7,1.4 \mathrm{~Hz}, 1 \mathrm{H}), 7.35-7.32(\mathrm{~m}, 2 \mathrm{H})$, $7.15(\mathrm{dd}, J=8.1,1.2 \mathrm{~Hz}, 1 \mathrm{H}), 7.10(\mathrm{ddd}, J=8.3,7.2,1.2 \mathrm{~Hz}$, $1 \mathrm{H}), 4.94(\mathrm{~d}, J=2.4 \mathrm{~Hz}, 1 \mathrm{H}), 3.43(\mathrm{~d}, J=2.5 \mathrm{~Hz}, 1 \mathrm{H}) .{ }^{13} \mathrm{C}$ NMR (126 MHz, DMSO- $\left.d_{6}\right) \delta 173.92,141.93,141.31,134.70$, $134.05,131.51,131.32,130.13,128.00,124.50,124.45,118.00$, $115.63,89.35$. HRMS (ESI) $m / z$ : calc. for $\mathrm{C}_{16} \mathrm{H}_{12} \mathrm{~N}_{3} \mathrm{~S}_{2}[\mathrm{M}+\mathrm{H}]^{+}$: 310.0467 , found 310.0421 .

\section{Synthesis of $41^{*}$}

A mixture of $4 \mathbf{l}(1 \mathrm{mmol})$ and ethylamine $(1.1 \mathrm{mmol})$ in dichloromethane $(5 \mathrm{ml})$ were refluxed for $4 \mathrm{~h}$. After cooling it down to the room temperature, the precipitate was filtered and washed with ethyl acetate. Recrystallization from ethanol produced yellow solid.

\section{1-Ethyl-3-(4-(4-methylene-2-thioxo-1,4-dihydroquinazolin-} $3(2 \mathrm{H})-y l)$ phenyl)thiourea $\left(4 l^{*}\right)$

Yellow solid. m.p. $197 \sim 202^{\circ} \mathrm{C}$; $1 \mathrm{H}$ NMR (400 MHz, DMSO$\left.\mathrm{d}_{6}\right) \delta 10.33(\mathrm{~s}, 1 \mathrm{H}), 9.69(\mathrm{~s}, 1 \mathrm{H}), 7.94(\mathrm{~s}, 1 \mathrm{H}), 7.67(\mathrm{~d}, \mathrm{~J}=$ $8.0 \mathrm{~Hz}, 1 \mathrm{H}), 7.61$ (d, J = 8.4 Hz, 2H), 7.42-7.33 (m, 1H), 7.16$7.07(\mathrm{~m}, 3 \mathrm{H}), 4.93(\mathrm{~d}, \mathrm{~J}=2.2 \mathrm{~Hz}, 1 \mathrm{H}), 3.55(\mathrm{~d}, \mathrm{~J}=2.1 \mathrm{~Hz}, 1 \mathrm{H})$, $3.50(\mathrm{q}, \mathrm{J}=6.6 \mathrm{~Hz}, 2 \mathrm{H}), 1.15(\mathrm{t}, \mathrm{J}=7.2 \mathrm{~Hz}, 3 \mathrm{H}) .13 \mathrm{C} \mathrm{NMR}$ (101 MHz, DMSO-d6) $\delta 180.40,174.19,142.15,139.56,137.43$, $134.11,131.21,129.62,124.45,124.38,121.66,118.04,115.51$, 89.45, 39.09, 14.61. HRMS (ESI) m/z: calc. for $\mathrm{C}_{18} \mathrm{H}_{18} \mathrm{~N}_{4} \mathrm{~S}_{2}[\mathrm{M}$ $+\mathrm{H}]^{+}: 355.1046$, found 355.1057 .

\section{Synthesis of $6 a$ and $6 r$}

A mixture of 2-aminoacetophenone $(1 \mathrm{mmol})$ and isocyanatobenzene/isocyanatocyclohexane $(1.1 \mathrm{mmol})$ in acetonitrile $(5 \mathrm{ml})$ was stirred at $82^{\circ} \mathrm{C}$ for $4 \mathrm{~h}$. After completion, the reaction solvent was reduced to half and upon cooling a white or yellow precipitate was obtained. Recrystallization from ethanol produced white solid.

\section{1-(2-Acetylphenyl)-3-phenylurea (6a)}

White solid. m.p. $218-221^{\circ} \mathrm{C}$ (Iwamura et al., 1980, 223 ${ }^{\circ} \mathrm{C}$ ); ${ }^{1} \mathrm{H}$ NMR (400 MHz, Chloroform-d) $\delta 11.34$ (s, 1H), 8.60 (dd, $J=$ $8.6,1.2 \mathrm{~Hz}, 1 \mathrm{H}), 7.85(\mathrm{dd}, J=8.1,1.6 \mathrm{~Hz}, 1 \mathrm{H}), 7.52(\mathrm{ddd}, J=8.6$, 7.1, 1.6 Hz, 1H), 7.48-7.39 (m, 2H), 7.38-7.29 (m, 2H), 7.16-7.08 (m, 1H), 7.03 (ddd, $J=8.3,7.2,1.2 \mathrm{~Hz}, 1 \mathrm{H}), 6.94(\mathrm{~s}, 1 \mathrm{H}), 2.63$ (s, 3H). ${ }^{13} \mathrm{C}$ NMR (101 MHz, Chloroform-d) $\delta$ 203.04, 152.81, $142.43,137.99,135.18,131.68,129.16,124.09,121.23,120.99$, 120.84, 120.19, 28.49. HRMS (ESI) m/z: calc. for $\mathrm{C}_{15} \mathrm{H}_{13} \mathrm{~N}_{2} \mathrm{O}_{2}[\mathrm{M}$ $+\mathrm{H}]^{+}: 255.1128$, found 255.1099 .

\section{1-(2-Acetylphenyl)-3-cyclohexylurea (6r)}

White solid. m.p. $175-178^{\circ} \mathrm{C}$; ${ }^{1} \mathrm{H}$ NMR (500 MHz, DMSO- $d_{6}$ ) $\delta 10.43(\mathrm{~s}, 1 \mathrm{H}), 8.38(\mathrm{dd}, J=8.6,1.2 \mathrm{~Hz}, 1 \mathrm{H}), 7.94(\mathrm{dd}, J=$ $8.0,1.6 \mathrm{~Hz}, 1 \mathrm{H}), 7.48$ (ddd, $J=8.7,7.1,1.6 \mathrm{~Hz}, 1 \mathrm{H}), 7.36$ (br s, $1 \mathrm{H}), 6.99$ (ddd, $J=8.2,7.2,1.2 \mathrm{~Hz}, 1 \mathrm{H}), 3.48-3.38(\mathrm{~m}, 1 \mathrm{H})$, 2.61 (s, 3H), 1.84-1.75 (m, 2H), 1.74-1.65 (m, 2H), 1.34-1.22 $(\mathrm{m}, 2 \mathrm{H}), 1.22-1.00(\mathrm{~m}, 4 \mathrm{H}) .{ }^{13} \mathrm{C}$ NMR $\left(126 \mathrm{MHz}, \mathrm{DMSO}-d_{6}\right)$ $\delta 202.59,154.47,142.67,134.55,132.40,121.79,120.34,119.91$, 48.74, 33.32, 29.16, 25.75, 25.15. HRMS (ESI) m/z: calc. for $\mathrm{C}_{15} \mathrm{H}_{20} \mathrm{~N}_{2} \mathrm{O}_{2}[\mathrm{M}+\mathrm{H}]^{+}: 261.1598$, found 261.1572.

\section{Synthesis of 7a-7ab}

A mixture of 2-aminoacetophenone 1 (1 $\mathrm{mmol})$, isocyanatobenzene $5(1.1 \mathrm{mmol})$ and a drop of aqueous $\mathrm{NaOH}$ solution $(5 \mathrm{M}, 10 \mathrm{~mol} \%)$ in acetonitrile $(5 \mathrm{ml})$ were stirred at room temperature. After completion, the reaction solvent was reduced to half and upon cooling a white or yellow precipitate was obtained. Recrystallization from ethanol produced white solid.

\section{4-Methylene-3-phenyl-3,4-dihydroquinazolin-2(1H)-} one (7a)

White solid. m.p. $186 \sim 188^{\circ} \mathrm{C}$ (Sbei et al., 2018, $\left.188 \sim 190^{\circ} \mathrm{C}\right) ;{ }^{1} \mathrm{H}$ NMR (400 MHz, Chloroform-d) $\delta 8.18(\mathrm{~s}, 1 \mathrm{H}), 7.59-7.49(\mathrm{~m}$, $3 \mathrm{H}), 7.47-7.40(\mathrm{~m}, 1 \mathrm{H}), 7.35-7.28(\mathrm{~m}, 2 \mathrm{H}), 7.24(\mathrm{dd}, J=7.9$, $1.3 \mathrm{~Hz}, 1 \mathrm{H}), 7.06-6.96(\mathrm{~m}, 1 \mathrm{H}), 6.72(\mathrm{dd}, J=8.1,1.2 \mathrm{~Hz}, 1 \mathrm{H}), 4.74$ $(\mathrm{d}, J=2.2 \mathrm{~Hz}, 1 \mathrm{H}), 3.68(\mathrm{~d}, J=2.2 \mathrm{~Hz}, 1 \mathrm{H}) .{ }^{13} \mathrm{C} \mathrm{NMR}(101 \mathrm{MHz}$, Chloroform-d) $\delta 150.88,143.31,138.42,135.16,130.27,129.94$, $129.19,128.34,123.94,122.73,117.03,115.09,87.43$. HRMS (ESI) $m / z$ : calc. for $\mathrm{C}_{15} \mathrm{H}_{12} \mathrm{~N}_{2} \mathrm{O}[\mathrm{M}+\mathrm{H}]^{+}: 237.1022$, found 237.1908 .

\section{4-Methylene-3-(o-tolyl)-3,4-dihydroquinazolin-2(1H)- one (7b)}

White solid. m.p. 204 207 ${ }^{\circ}$; ${ }^{1} \mathrm{H}$ NMR (500 MHz, DMSO- $d_{6}$ ) $\delta 10.30(\mathrm{~s}, 1 \mathrm{H}), 7.68(\mathrm{dd}, J=8.1,1.3 \mathrm{~Hz}, 1 \mathrm{H}), 7.42-7.37(\mathrm{~m}$, $1 \mathrm{H}), 7.37-7.30(\mathrm{~m}, 3 \mathrm{H}), 7.19-7.15(\mathrm{~m}, 1 \mathrm{H}), 7.01(\mathrm{td}, J=7.8$, $1.2 \mathrm{~Hz}, 1 \mathrm{H}), 6.96(\mathrm{dd}, J=8.1,1.2 \mathrm{~Hz}, 1 \mathrm{H}), 4.75(\mathrm{~d}, J=1.8 \mathrm{~Hz}$, $1 \mathrm{H}), 3.33(\mathrm{~d}, J=1.8 \mathrm{~Hz}, 1 \mathrm{H}), 2.11(\mathrm{~s}, 3 \mathrm{H}),{ }^{13} \mathrm{C}$ NMR $(126$ $\left.\mathrm{MHz}, \mathrm{DMSO}-d_{6}\right) \delta 149.27,142.44,137.75,136.56,136.42,131.50$, $130.90,129.77,128.77,127.89,124.63,122.61,116.29,115.29$, 85.21, 17.19. HRMS (ESI) $m / z$ : calc. for $\mathrm{C}_{16} \mathrm{H}_{14} \mathrm{~N}_{2} \mathrm{O}[\mathrm{M}+\mathrm{H}]^{+}$: 251.1179, found 251.1121. 
4-Methylene-3-(m-tolyl)-3,4-dihydroquinazolin-2(1H)one (7c)

Yellow powder. m.p. 206 208 ${ }^{\circ} \mathrm{C}$; ${ }^{1} \mathrm{H}$ NMR (500 MHz, DMSO$\left.d_{6}\right) \delta 10.27(\mathrm{~s}, 1 \mathrm{H}), 7.65(\mathrm{dd}, J=8.0,1.3 \mathrm{~Hz}, 1 \mathrm{H}), 7.40(\mathrm{t}, J$ $=7.7 \mathrm{~Hz}, 1 \mathrm{H}), 7.31(\mathrm{ddd}, J=8.4,6.0,1.4 \mathrm{~Hz}, 1 \mathrm{H}), 7.25-7.22$ $(\mathrm{m}, 1 \mathrm{H}), 7.09-7.03(\mathrm{~m}, 2 \mathrm{H}), 6.99$ (ddd, $J=8.3,7.2,1.2 \mathrm{~Hz}$, $1 \mathrm{H}), 6.95(\mathrm{dd}, J=8.1,1.2 \mathrm{~Hz}, 1 \mathrm{H}), 4.76(\mathrm{~d}, J=1.8 \mathrm{~Hz}, 1 \mathrm{H})$, $3.43(\mathrm{~d}, J=1.8 \mathrm{~Hz}, 1 \mathrm{H}), 2.35(\mathrm{~s}, 3 \mathrm{H}) .{ }^{13} \mathrm{C} \mathrm{NMR}(126 \mathrm{MHz}$, DMSO- $\left.d_{6}\right) \delta 149.71,143.72,139.63,139.04,136.37,130.82$, $130.16,129.92,129.07,126.71,124.53,122.57,116.59,115.22$, 86.38, 21.24. HRMS (ESI) $m / z$ : calc. for $\mathrm{C}_{16} \mathrm{H}_{14} \mathrm{~N}_{2} \mathrm{O}[\mathrm{M}+\mathrm{H}]^{+}$: 251.1179, found 251.1098.

\section{4-Methylene-3-(p-tolyl)-3,4-dihydroquinazolin-2(1H)- one (7d)}

Yellow powder. m.p. 222 224 ${ }^{\circ} \mathrm{C} ;{ }^{1} \mathrm{H}$ NMR (500 MHz, DMSO$\left.d_{6}\right) \delta 10.26(\mathrm{~s}, 1 \mathrm{H}), 7.66(\mathrm{dd}, J=8.1,1.3 \mathrm{~Hz}, 1 \mathrm{H}), 7.36(\mathrm{~d}, J=$ $8.4 \mathrm{~Hz}, 1 \mathrm{H}), 7.31(\mathrm{td}, J=8.0 \mathrm{~Hz}, 1.2 \mathrm{~Hz}, 1 \mathrm{H}), 7.15-7.11(\mathrm{~m}, 2 \mathrm{H})$, $7.05(\mathrm{~d}, J=8.3 \mathrm{~Hz}, 1 \mathrm{H}), 7.00(\mathrm{td}, J=8.1 \mathrm{~Hz}, 1.2 \mathrm{~Hz}, 1 \mathrm{H}), 6.94(\mathrm{~d}$, $J=7.9 \mathrm{~Hz}, 1 \mathrm{H}), 4.76(\mathrm{~d}, J=1.8 \mathrm{~Hz}, 1 \mathrm{H}), 3.43(\mathrm{~d}, J=1.8 \mathrm{~Hz}, 1 \mathrm{H})$, $2.37(\mathrm{~s}, 3 \mathrm{H}) .{ }^{13} \mathrm{C}$ NMR $\left(126 \mathrm{MHz}, \mathrm{DMSO}-d_{6}\right) \delta 149.77,143.81$, $138.18,137.74,136.49,136.36,130.83,130.65,129.49,124.56$, $122.56,118.61,116.58,115.20,86.25,21.21$. HRMS (ESI) $\mathrm{m} / z$ : calc. for $\mathrm{C}_{16} \mathrm{H}_{14} \mathrm{~N}_{2} \mathrm{O}[\mathrm{M}+\mathrm{H}]^{+}: 251.1179$, found 251.1093 .

\section{3-(2-Fluorophenyl)-4-methylene-3,4-dihydroquinazolin- 2(1H)-one (7e)}

Light yellow powder. m.p. 220 224 ${ }^{\circ} \mathrm{C} ;{ }^{1} \mathrm{H}$ NMR $(500 \mathrm{MHz}$, DMSO- $\left.d_{6}\right) \delta 10.42(\mathrm{~s}, 1 \mathrm{H}), 7.69(\mathrm{dd}, J=8.1,1.3 \mathrm{~Hz}, 1 \mathrm{H}), 7.55-$ $7.48(\mathrm{~m}, 1 \mathrm{H}), 7.46-7.39(\mathrm{~m}, 2 \mathrm{H}), 7.39-7.31(\mathrm{~m}, 2 \mathrm{H}), 7.03$ (ddd, $J=8.3,7.3,1.2 \mathrm{~Hz}, 1 \mathrm{H}), 6.97(\mathrm{dd}, J=8.1,1.3 \mathrm{~Hz}, 1 \mathrm{H}), 4.82(\mathrm{~d}$, $J=2.2 \mathrm{~Hz}, 1 \mathrm{H}), 3.48(\mathrm{dd}, J=2.2,0.8 \mathrm{~Hz}, 1 \mathrm{H}) .{ }^{13} \mathrm{C}$ NMR $(126$ $\left.\mathrm{MHz}, \mathrm{DMSO}-d_{6}\right) \delta 158.65(\mathrm{~d}, J=248 \mathrm{~Hz}), 149.25,142.67,136.14$, 132.07, 131.07, 130.98 (d, $J=7.6 \mathrm{~Hz}), 126.12(\mathrm{~d}, J=12.6 \mathrm{~Hz})$, $125.97(\mathrm{~d}, J=3.8 \mathrm{~Hz}), 124.66,122.85,117.15(\mathrm{~d}, J=18.9 \mathrm{~Hz})$, $116.21,115.41,85.80$. HRMS (ESI) $m / z$ : calc. for $\mathrm{C}_{15} \mathrm{H}_{11} \mathrm{FN}_{2} \mathrm{O}$ $[\mathrm{M}+\mathrm{H}]^{+}: 255.0928$, found 255.0876 .

\section{3-(3-Fluorophenyl)-4-methylene-3,4-dihydroquinazolin- 2(1H)-one $(7 f)$}

Brown powder. m.p. $228 \sim 230^{\circ} \mathrm{C} ;{ }^{1} \mathrm{H}$ NMR $(600 \mathrm{MHz}$, DMSO$\left.d_{6}\right) \delta 10.37(\mathrm{~s}, 1 \mathrm{H}), 7.68(\mathrm{dd}, J=8.1,1.3 \mathrm{~Hz}, 1 \mathrm{H}), 7.60-7.54(\mathrm{~m}$, $1 \mathrm{H}), 7.35-7.27(\mathrm{~m}, 2 \mathrm{H}), 7.24(\mathrm{dt}, J=9.7,2.2 \mathrm{~Hz}, 1 \mathrm{H}), 7.15(\mathrm{dd}$, $J=7.8,1.0 \mathrm{~Hz}, 1 \mathrm{H}), 7.01(\mathrm{ddd}, J=8.3,7.4,1.2 \mathrm{~Hz}, 1 \mathrm{H}), 6.95$ $(\mathrm{dd}, J=8.1,1.2 \mathrm{~Hz}, 1 \mathrm{H}), 4.81(\mathrm{~d}, J=2.1 \mathrm{~Hz}, 1 \mathrm{H}), 3.44(\mathrm{~d}, J=$ $2.1 \mathrm{~Hz}, 1 \mathrm{H}) .{ }^{13} \mathrm{C}$ NMR $\left(151 \mathrm{MHz}, \mathrm{DMSO}-d_{6}\right) \delta 163.18(\mathrm{~d}, \mathrm{~J}=$ $245 \mathrm{~Hz}), 149.52,143.48,140.70(\mathrm{~d}, J=9.1 \mathrm{~Hz}), 136.23,131.64(\mathrm{~d}$, $J=9.1 \mathrm{~Hz}), 130.93,126.23(\mathrm{~d}, J=3.0 \mathrm{~Hz}), 124.57,122.67,117.32$ $(\mathrm{d}, J=22.7 \mathrm{~Hz}), 116.51,115.56(\mathrm{~d}, J=21 \mathrm{~Hz}), 115.28,86.47$. HRMS (ESI) $m / z$ : calc. for $\mathrm{C}_{15} \mathrm{H}_{11} \mathrm{FN}_{2} \mathrm{O}[\mathrm{M}+\mathrm{H}]^{+}:$255.0928, found 255.0885 .

\section{3-(4-Fluorophenyl)-4-methylene-3,4-dihydroquinazolin- 2(1H)-one $(7 g)$}

Light yellow powder. m.p. $245 \sim 247^{\circ} \mathrm{C}$ (Molina et al., 1993, $\left.136 \sim 138^{\circ} \mathrm{C}\right) ;{ }^{1} \mathrm{H}$ NMR $\left(500 \mathrm{MHz}, \mathrm{DMSO}-d_{6}\right) \delta 10.33(\mathrm{~s}, 1 \mathrm{H})$, $7.67(\mathrm{dd}, J=8.1,1.3 \mathrm{~Hz}, 1 \mathrm{H}), 7.36-7.30(\mathrm{~m}, 5 \mathrm{H}), 7.01$ (ddd,
$J=8.2,7.3,1.2 \mathrm{~Hz}, 1 \mathrm{H}), 6.95(\mathrm{dd}, J=8.1,1.2 \mathrm{~Hz}, 1 \mathrm{H}), 4.79$ $(\mathrm{d}, J=2.0 \mathrm{~Hz}, 1 \mathrm{H}), 3.42(\mathrm{~d}, J=2.0 \mathrm{~Hz}, 1 \mathrm{H}) .{ }^{13} \mathrm{C}$ NMR $(126$ $\left.\mathrm{MHz}, \mathrm{DMSO}-d_{6}\right) \delta 161.80(\mathrm{~d}, J=246 \mathrm{~Hz}), 149.74,143.82$, $136.29,135.31(\mathrm{~d}, J=2.5 \mathrm{~Hz}), 131.97(\mathrm{~d}, J=8.8 \mathrm{~Hz}), 130.90$, $124.58,122.63,117.02$ (d, $J=22.7 \mathrm{~Hz}), 116.54,115.27,86.38$. HRMS (ESI) $m / z$ : calc. for $\mathrm{C}_{15} \mathrm{H}_{11} \mathrm{FN}_{2} \mathrm{O}[\mathrm{M}+\mathrm{H}]^{+}:$255.0928, found 255.0943.

\section{3-(2-Chlorophenyl)-4-methylene-3,4-dihydroquinazolin- 2(1H)-one (7h)}

Light yellow powder. m.p. $229 \sim 231^{\circ} \mathrm{C} ;{ }^{1} \mathrm{H}$ NMR $(400 \mathrm{MHz}$, DMSO- $\left.d_{6}\right) \delta 10.53(\mathrm{~s}, 1 \mathrm{H}), 7.71(\mathrm{ddd}, J=9.3,7.2,1.9 \mathrm{~Hz}, 2 \mathrm{H})$, $7.58-7.44(\mathrm{~m}, 3 \mathrm{H}), 7.36$ (ddd, $J=8.3,7.4,1.3 \mathrm{~Hz}, 1 \mathrm{H}), 7.09-$ $6.98(\mathrm{~m}, 2 \mathrm{H}), 4.82(\mathrm{~d}, J=2.2 \mathrm{~Hz}, 1 \mathrm{H}), 3.35(\mathrm{~d}, J=2.2 \mathrm{~Hz}, 1 \mathrm{H})$. ${ }^{13} \mathrm{C}$ NMR $\left(101 \mathrm{MHz}\right.$, DMSO- $\left.d_{6}\right) \delta 149.10,142.19,136.30,136.22$, $133.08,132.16,131.00,130.84,130.55,129.17,124.64,122.75$, $116.26,115.47,85.50$. HRMS (ESI) $m / z$ : calc. for $\mathrm{C}_{15} \mathrm{H}_{11} \mathrm{ClN}_{2} \mathrm{O}$ $[\mathrm{M}+\mathrm{H}]^{+}: 271.0633$, found 271.0598 .

\section{3-(3-Chlorophenyl)-4-methylene-3,4-dihydroquinazolin- 2(1H)-one (7i)}

White powder. m.p. $227 \sim 229^{\circ} \mathrm{C}$; ${ }^{1} \mathrm{H}$ NMR (400 MHz, DMSO$\left.d_{6}\right) \delta 10.46(\mathrm{~s}, 1 \mathrm{H}), 7.70(\mathrm{dd}, J=8.1,1.3 \mathrm{~Hz}, 1 \mathrm{H}), 7.63-7.51(\mathrm{~m}$, $2 \mathrm{H}), 7.46(\mathrm{t}, J=2.0 \mathrm{~Hz}, 1 \mathrm{H}), 7.39-7.29(\mathrm{~m}, 2 \mathrm{H}), 7.08-6.95(\mathrm{~m}$, $2 \mathrm{H}), 4.84(\mathrm{~d}, J=2.1 \mathrm{~Hz}, 1 \mathrm{H}), 3.44(\mathrm{~d}, J=2.1 \mathrm{~Hz}, 1 \mathrm{H}) .{ }^{13} \mathrm{C} \mathrm{NMR}$ $\left(101 \mathrm{MHz}, \mathrm{DMSO}-d_{6}\right) \delta$ 149.57, 143.58, 140.56, 136.28, 134.12, $131.75,130.95,130.12,128.91,128.67,124.58,122.69,116.54$, $115.36,86.54$. HRMS (ESI) $m / z$ : calc. for $\mathrm{C}_{15} \mathrm{H}_{11} \mathrm{ClN}_{2} \mathrm{O}[\mathrm{M}+$ $\mathrm{H}]^{+}: 271.0633$, found 271.0569 .

\section{3-(4-Chlorophenyl)-4-methylene-3,4-dihydroquinazolin- 2(1H)-one $(7 j)$}

Light green powder. m.p. $223 \sim 225^{\circ} \mathrm{C}$ (Sbei et al., 2018, $214 \sim 217^{\circ} \mathrm{C}$ ); ${ }^{1} \mathrm{H}$ NMR (400 MHz, DMSO-d $)_{6} \delta 10.42(\mathrm{~s}, 1 \mathrm{H})$, $7.70(\mathrm{dd}, J=8.1,1.3 \mathrm{~Hz}, 1 \mathrm{H}), 7.64-7.57$ (m, 2H), 7.40-7.31 (m, $3 \mathrm{H}), 7.08-6.96(\mathrm{~m}, 2 \mathrm{H}), 4.83(\mathrm{~d}, J=2.1 \mathrm{~Hz}, 1 \mathrm{H}), 3.45(\mathrm{~d}, J=$ $2.1 \mathrm{~Hz}, 1 \mathrm{H}) .{ }^{13} \mathrm{C}$ NMR $\left(101 \mathrm{MHz}, \mathrm{DMSO}-d_{6}\right) \delta 149.61,143.63$, $138.05,136.29,133.00,131.90,130.93,130.27,124.58,122.67$, $116.54,115.33,86.49$. HRMS (ESI) $m / z$ : calc. for $\mathrm{C}_{15} \mathrm{H}_{11} \mathrm{ClN}_{2} \mathrm{O}$ $[\mathrm{M}+\mathrm{H}]^{+}: 271.0633$, found 271.0896 .

\section{4-Methylene-3-(2-(trifluoromethyl)phenyl)-3,4- dihydroquinazolin-2(1H)-one (7k)}

Yellow solid. m.p. $309 \sim 312^{\circ} \mathrm{C} ;{ }^{1} \mathrm{H}$ NMR (500 MHz, DMSO- $d_{6}$ ) $\delta 10.42(\mathrm{~s}, 1 \mathrm{H}), 7.91(\mathrm{dd}, J=7.9,1.5 \mathrm{~Hz}, 1 \mathrm{H}), 7.86(\mathrm{td}, J=7.8$, $1.5 \mathrm{~Hz}, 1 \mathrm{H}), 7.70(\mathrm{td}, J=8.5,1.4 \mathrm{~Hz}, 2 \mathrm{H}), 7.53(\mathrm{~d}, J=7.8 \mathrm{~Hz}$, $1 \mathrm{H}$ ), 7.34 (ddd, $J=7.8,7.3,1.3 \mathrm{~Hz}, 1 \mathrm{H}$ ), 7.02 (ddd, $J=7.8,7.4$, $1.3 \mathrm{~Hz}, 1 \mathrm{H}), 6.97(\mathrm{dd}, J=8.1,1.3 \mathrm{~Hz}, 1 \mathrm{H}), 4.84(\mathrm{~d}, J=2.3 \mathrm{~Hz}$, $1 \mathrm{H}), 3.26(\mathrm{~d}, J=2.4 \mathrm{~Hz}, 1 \mathrm{H}) .{ }^{13} \mathrm{C}$ NMR $\left(126 \mathrm{MHz}, \mathrm{DMSO}-d_{6}\right)$ $\delta 149.53,143.52,136.70,136.12,134.73,133.11,130.98,129.79$, 128.16 (m), 127.83, 124.87, 124.53, 122.76, 116.34, 115.40, 86.66. HRMS (ESI) $m / z$ : calc. for $\mathrm{C}_{16} \mathrm{H}_{12} \mathrm{~F}_{3} \mathrm{~N}_{2} \mathrm{O}[\mathrm{M}+\mathrm{H}]^{+}: 305.0896$, found 305.0822 .

\section{3-Mesityl-4-methylene-3,4-dihydroquinazolin-2(1H)- one ( $7 l)$}

White powder. m.p. $230 \sim 232^{\circ} \mathrm{C} ;{ }^{1} \mathrm{H}$ NMR (500 MHz, DMSO$\left.d_{6}\right) \delta 10.31(\mathrm{~s}, 1 \mathrm{H}), 7.69(\mathrm{dd}, J=8.1,1.3 \mathrm{~Hz}, 1 \mathrm{H}), 7.32(\mathrm{ddd}, J=$ 
8.3, 7.3, 1.3 Hz, 1H), $7.00(\mathrm{~d}, J=4.7 \mathrm{~Hz}, 3 \mathrm{H}), 6.99-6.95(\mathrm{~m}, 1 \mathrm{H})$, $4.70(\mathrm{~d}, J=1.7 \mathrm{~Hz}, 1 \mathrm{H}), 3.37(\mathrm{~d}, J=1.6 \mathrm{~Hz}, 1 \mathrm{H}), 2.29(\mathrm{~s}, 3 \mathrm{H})$, $2.04(\mathrm{~s}, 6 \mathrm{H}) .{ }^{13} \mathrm{C}$ NMR $\left(126 \mathrm{MHz}, \mathrm{DMSO}-d_{6}\right) \delta 148.95,141.08$, $137.57,136.51,135.94,133.93,130.90,129.66,124.71,122.59$, $116.03,115.32,83.80,21.07,17.37$. HRMS (ESI) $m / z$ : calc. for $\mathrm{C}_{18} \mathrm{H}_{18} \mathrm{~N}_{2} \mathrm{O}[\mathrm{M}+\mathrm{H}]^{+}:$279.1492, found 279.1421.

\section{3-(2,6-Diethylphenyl)-4-methylene-3,4-dihydroquinazolin- 2(1H)-one (7m)}

White solid. m.p. $166 \sim 168^{\circ} \mathrm{C} ;{ }^{1} \mathrm{H}$ NMR $\left(500 \mathrm{MHz}, \mathrm{DMSO}-d_{6}\right) \delta$ $10.33(\mathrm{~s}, 1 \mathrm{H}), 7.69(\mathrm{dd}, J=8.2,1.3 \mathrm{~Hz}, 1 \mathrm{H}), 7.39-7.29(\mathrm{~m}, 2 \mathrm{H})$, $7.25(\mathrm{~d}, J=7.6 \mathrm{~Hz}, 2 \mathrm{H}), 7.01(\mathrm{ddd}, J=8.3,7.3,1.2 \mathrm{~Hz}, 1 \mathrm{H}), 6.97$ $(\mathrm{dd}, J=8.1,1.2 \mathrm{~Hz}, 1 \mathrm{H}), 4.74(\mathrm{~d}, J=1.7 \mathrm{~Hz}, 1 \mathrm{H}), 3.31(\mathrm{~d}, J=$ $1.8 \mathrm{~Hz}, 1 \mathrm{H}), 2.42(\mathrm{q}, J=7.5 \mathrm{~Hz}, 4 \mathrm{H}), 1.12(\mathrm{t}, J=7.6 \mathrm{~Hz}, 6 \mathrm{H})$. ${ }^{13} \mathrm{C}$ NMR $\left(126 \mathrm{MHz}, \mathrm{DMSO}-d_{6}\right) \delta 149.43,142.12,141.77,136.44$, $135.36,132.56,130.97,128.88,127.15,124.68,122.66,120.86$, $116.00,115.32,84.83,24.84,23.79,15.07,14.50$. HRMS (ESI) $m / z$ : calc. for $\mathrm{C}_{19} \mathrm{H}_{20} \mathrm{~N}_{2} \mathrm{O}[\mathrm{M}+\mathrm{H}]^{+}$: 293.1648, found 293.1606.

\section{3-(2,4-Difluorophenyl)-4-methylene-3,4-dihydroquinazolin- 2(1H)-one (7n)}

White powder. m.p. $226 \sim 228^{\circ} \mathrm{C}$; ${ }^{1} \mathrm{H}$ NMR $(500 \mathrm{MHz}$, DMSO$\left.d_{6}\right) \delta 10.46(\mathrm{~s}, 1 \mathrm{H}), 7.70(\mathrm{dd}, J=8.0,1.3 \mathrm{~Hz}, 1 \mathrm{H}), 7.55-7.47$ (m, 2H), 7.34 (ddd, $J=8.4,7.3,1.3 \mathrm{~Hz}, 1 \mathrm{H}), 7.28-7.22(\mathrm{~m}, 1 \mathrm{H})$, 7.03 (ddd, $J=8.2,7.3,1.2 \mathrm{~Hz}, 1 \mathrm{H}), 6.97(\mathrm{dd}, J=8.1,1.2 \mathrm{~Hz}$, $1 \mathrm{H}), 4.84(\mathrm{~d}, J=2.4 \mathrm{~Hz}, 1 \mathrm{H}), 3.52(\mathrm{~d}, J=2.3 \mathrm{~Hz}, 1 \mathrm{H}) .{ }^{13} \mathrm{C}$ NMR $\left(126 \mathrm{MHz}, \mathrm{DMSO}-d_{6}\right) \delta 162.30(\mathrm{dd}, J=247.5,11.8 \mathrm{~Hz})$, $158.86(\mathrm{dd}, J=251.1,13.2 \mathrm{~Hz}), 149.26,142.64,136.08,133.28$ $(\mathrm{dd}, J=9.9,2.1 \mathrm{~Hz}), 131.11,124.69,122.89,122.70(\mathrm{dd}, J=13.4$, $4.0 \mathrm{~Hz}), 116.18,115.44,113.07(\mathrm{dd}, J=22.5,3.6 \mathrm{~Hz}), 105.78(\mathrm{dd}$, $J=27.1,24.1 \mathrm{~Hz}), 85.90$. HRMS (ESI) $\mathrm{m} / z$ : $\left(\mathrm{M}+\mathrm{H}^{+}\right)$; calc. for $\mathrm{C}_{15} \mathrm{H}_{10} \mathrm{~F}_{2} \mathrm{~N}_{2} \mathrm{O}[\mathrm{M}+\mathrm{H}]^{+}: 273.0834$, found 273.0789.

\section{3-(3,4-Dichlorophenyl)-4-methylene-3,4-dihydroquinazolin- 2(1H)-one (7o)}

White powder. m.p. $234 \sim 236^{\circ} \mathrm{C}$; ${ }^{1} \mathrm{H}$ NMR $(400 \mathrm{MHz}$, DMSO$\left.d_{6}\right) \delta 10.45(\mathrm{~s}, 1 \mathrm{H}), 7.82(\mathrm{dd}, J=8.5,1.2 \mathrm{~Hz}, 1 \mathrm{H}), 7.76-7.67(\mathrm{~m}$, $2 \mathrm{H}), 7.41-7.31(\mathrm{~m}, 2 \mathrm{H}), 7.09-7.00(\mathrm{~m}, 1 \mathrm{H}), 6.98(\mathrm{dd}, J=8.1$, $1.2 \mathrm{~Hz}, 1 \mathrm{H}), 4.85(\mathrm{~d}, J=2.3 \mathrm{~Hz}, 1 \mathrm{H}), 3.49(\mathrm{~d}, J=2.3 \mathrm{~Hz}, 1 \mathrm{H})$. ${ }^{13} \mathrm{C}$ NMR $\left(101 \mathrm{MHz}\right.$, DMSO- $\left.d_{6}\right) \delta 149.53,143.45,139.15,136.28$, $132.36,132.08,131.33,130.97,130.67,124.59,122.71,116.53$, 115.41, 86.71. HRMS (ESI) $m / z$ : calc. for $\mathrm{C}_{15} \mathrm{H}_{10} \mathrm{Cl}_{2} \mathrm{~N}_{2} \mathrm{O}[\mathrm{M}+$ $\mathrm{H}]^{+}:$305.0243, found 305.0185.

\section{3-Benzyl-4-methylene-3,4-dihydroquinazolin-2(1H)- one (7p)}

White solid. m.p. $212 \sim 214^{\circ} \mathrm{C} ;{ }^{1} \mathrm{H}$ NMR (500 MHz, DMSO- $d_{6}$ ) $\delta 10.28(\mathrm{~s}, 1 \mathrm{H}), 7.62(\mathrm{~d}, J=8.1 \mathrm{~Hz}, 1 \mathrm{H}), 7.36-7.19(\mathrm{~m}, 6 \mathrm{H})$, $7.00-6.92(\mathrm{~m}, 2 \mathrm{H}), 5.01(\mathrm{~s}, 2 \mathrm{H}), 4.82(\mathrm{~d}, J=2.4 \mathrm{~Hz}, 1 \mathrm{H}), 4.12$ $(\mathrm{d}, J=2.5 \mathrm{~Hz}, 1 \mathrm{H}) .{ }^{13} \mathrm{C}$ NMR $\left(126 \mathrm{MHz}, \mathrm{DMSO}-d_{6}\right) \delta 150.60$, $140.23,137.50,136.08,130.65,128.92,127.21,126.81,124.40$, $122.62,116.46,115.12,85.70,46.30$. HRMS (ESI) $\mathrm{m} / z$ : calc. for $\mathrm{C}_{16} \mathrm{H}_{14} \mathrm{~N}_{2} \mathrm{O}[\mathrm{M}+\mathrm{H}]^{+}: 251.1179$, found 251.1085.

\section{4-Methylene-3-propyl-3,4-dihydroquinazolin-2(1H)- one $(7 q)$}

Brown powder. m.p. $114 \sim 117^{\circ} \mathrm{C}$; ${ }^{1} \mathrm{H}$ NMR (500 MHz, DMSO$\left.d_{6}\right) \delta 10.06(\mathrm{~s}, 1 \mathrm{H}), 7.65(\mathrm{dd}, J=8.2,1.3 \mathrm{~Hz}, 1 \mathrm{H}), 7.26$ (ddd,
$J=8.4,7.2,1.3 \mathrm{~Hz}, 1 \mathrm{H}), 6.96(\mathrm{ddd}, J=8.2,7.3,1.3 \mathrm{~Hz}, 1 \mathrm{H})$, $6.87(\mathrm{dd}, J=8.1,1.2 \mathrm{~Hz}, 1 \mathrm{H}), 4.87(\mathrm{~d}, J=2.3 \mathrm{~Hz}, 1 \mathrm{H}), 4.28$ $(\mathrm{d}, J=2.4 \mathrm{~Hz}, 1 \mathrm{H}), 3.70(\mathrm{t}, J=7.6 \mathrm{~Hz}, 2 \mathrm{H}), 1.65-1.55(\mathrm{~m}$, $2 \mathrm{H}), 0.89(\mathrm{t}, J=7.4 \mathrm{~Hz}, 3 \mathrm{H}) .{ }^{13} \mathrm{C}$ NMR $\left(126 \mathrm{MHz}, \mathrm{DMSO}-d_{6}\right)$ $\delta 150.07,140.25,136.15,130.51,124.45,122.41,116.51,114.90$, 84.02, 44.26, 18.91, 11.57. HRMS (ESI) $m / z$ : calc. for $\mathrm{C}_{12} \mathrm{H}_{14} \mathrm{~N}_{2} \mathrm{~S}$ $[\mathrm{M}+\mathrm{H}]^{+}: 203.1179$, found 203.1089.

\section{3-Cyclohexyl-4-methylene-3,4-dihydroquinazolin-2(1H)-} one $(7 r)$

White powder. m.p. $138 \sim 140^{\circ} \mathrm{C} ;{ }^{1} \mathrm{H}$ NMR (400 MHz, DMSO$\left.d_{6}\right) \delta 9.90(\mathrm{~s}, 1 \mathrm{H}), 7.58(\mathrm{dd}, J=8.0,1.3 \mathrm{~Hz}, 1 \mathrm{H}), 7.25(\mathrm{td}, J=$ $7.6,1.3 \mathrm{~Hz}, 1 \mathrm{H}), 7.01-6.89(\mathrm{~m}, 1 \mathrm{H}), 6.87(\mathrm{dd}, J=8.1,1.2 \mathrm{~Hz}$, $1 \mathrm{H}), 4.89(\mathrm{~d}, J=2.4 \mathrm{~Hz}, 1 \mathrm{H}), 4.53(\mathrm{~d}, J=2.4 \mathrm{~Hz}, 1 \mathrm{H}), 3.84(\mathrm{tt}$, $J=12.0,3.6 \mathrm{~Hz}, 1 \mathrm{H}), 2.39(\mathrm{qd}, J=12.5,3.6 \mathrm{~Hz}, 2 \mathrm{H}), 1.82-1.62$ (m, 4H), $1.45-1.01(\mathrm{~m}, 4 \mathrm{H}) .{ }^{13} \mathrm{C}$ NMR (101 MHz, DMSO- $\left.d_{6}\right)$ $\delta 150.56,142.08,136.27,130.15,124.40,122.27,118.46,114.34$, $88.15,58.37,29.02,26.42,25.70$. HRMS (ESI) $\mathrm{m} / z$ : calc. for $\mathrm{C}_{15} \mathrm{H}_{18} \mathrm{~N}_{2} \mathrm{O}[\mathrm{M}+\mathrm{H}]^{+}: 243.1492$, found 243.1464.

3-(4-Isocyanatophenyl)-4-methylene-3,4-dihydroquinazolin2(1H)-one (7s)

Light yellow powder. m.p. $230 \sim 232^{\circ} \mathrm{C} ;{ }^{1} \mathrm{H}$ NMR $(500 \mathrm{MHz}$, DMSO- $\left.d_{6}\right) \delta 11.75(\mathrm{~s}, 1 \mathrm{H}), 7.69(\mathrm{dd}, J=8.1,1.4 \mathrm{~Hz}, 1 \mathrm{H}), 7.57$ $(\mathrm{t}, J=7.9 \mathrm{~Hz}, 1 \mathrm{H}), 7.53-7.49(\mathrm{~m}, 1 \mathrm{H}), 7.43-7.34(\mathrm{~m}, 2 \mathrm{H}), 7.28-$ $7.22(\mathrm{~m}, 1 \mathrm{H}), 7.15(\mathrm{dd}, J=8.1,1.3 \mathrm{~Hz}, 1 \mathrm{H}), 7.13-7.08(\mathrm{~m}, 1 \mathrm{H})$, $4.95(\mathrm{~d}, J=2.5 \mathrm{~Hz}, 1 \mathrm{H}), 3.44(\mathrm{~d}, J=2.5 \mathrm{~Hz}, 1 \mathrm{H}) .{ }^{13} \mathrm{C} \mathrm{NMR}$ $\left(126 \mathrm{MHz}, \mathrm{DMSO}-d_{6}\right) \delta 173.93,143.32,141.91,134.18,134.05$, $131.85,131.30,129.90,128.81,128.79,124.49,124.43,118.03$, $115.63,89.34$. HRMS (ESI) $m / z$ : calc. for $\mathrm{C}_{16} \mathrm{H}_{11} \mathrm{~N}_{3} \mathrm{O}_{2}[\mathrm{M}+\mathrm{H}]^{+}$: 278.0924, found 278.0882 .

\section{6-Chloro-3-(2-fluorophenyl)-4-methylene-3,4- dihydroquinazolin-2(1H)-one $(7 t)$}

White powder. m.p. 258.9 261.3 ${ }^{\circ} \mathrm{C}$; ${ }^{1} \mathrm{H}$ NMR $(400 \mathrm{MHz}$, DMSO- $\left.d_{6}\right) \delta 10.69(\mathrm{~s}, 1 \mathrm{H}), 7.80(\mathrm{~d}, J=2.3 \mathrm{~Hz}, 1 \mathrm{H}), 7.56-7.48$ $(\mathrm{m}, 1 \mathrm{H}), 7.47-7.32(\mathrm{~m}, 4 \mathrm{H}), 6.99(\mathrm{dd}, J=8.7,1.9 \mathrm{~Hz}, 1 \mathrm{H}), 4.94$ $(\mathrm{d}, J=2.4 \mathrm{~Hz}, 1 \mathrm{H}), 3.52(\mathrm{~d}, J=2.5 \mathrm{~Hz}, 1 \mathrm{H}) .{ }^{13} \mathrm{C} \mathrm{NMR}(101 \mathrm{MHz}$, DMSO- $\left.d_{6}\right) \delta 158.58(\mathrm{~d}, J=249.0 \mathrm{~Hz}), 149.05,141.55,135.32$, $132.01,131.08(\mathrm{~d}, J=8.0 \mathrm{~Hz}), 130.87,126.79,126.01(\mathrm{~d}, J=$ $3.9 \mathrm{~Hz}), 125.86,124.15,117.97,117.32(\mathrm{~d}, J=9.7 \mathrm{~Hz}), 117.08$, 87.28. HRMS (ESI) $m / z$ : calc. for $\mathrm{C}_{16} \mathrm{H}_{14} \mathrm{FN}_{2} \mathrm{O}^{+}[\mathrm{M}+\mathrm{H}]^{+}$: 289.0538 , found 289.0552 .

\section{6-Chloro-3-(3-chlorophenyl)-4-methylene-3,4- dihydroquinazolin-2(1H)-one (7u)}

White powder. m.p. $245.6 \sim 247.2^{\circ} \mathrm{C} ;{ }^{1} \mathrm{H}$ NMR (400 MHz, DMSO$\left.d_{6}\right) \delta 10.53(\mathrm{~s}, 1 \mathrm{H}), 7.78(\mathrm{~d}, J=2.3 \mathrm{~Hz}, 1 \mathrm{H}), 7.61-7.48(\mathrm{~m}$, $2 \mathrm{H}), 7.45(\mathrm{t}, J=1.9 \mathrm{~Hz}, 1 \mathrm{H}), 7.38(\mathrm{dd}, J=8.6,2.3 \mathrm{~Hz}, 1 \mathrm{H})$, $7.29(\mathrm{dt}, J=7.6,1.7 \mathrm{~Hz}, 1 \mathrm{H}), 6.96(\mathrm{~d}, J=8.6 \mathrm{~Hz}, 1 \mathrm{H}), 4.94$ $(\mathrm{d}, J=2.4 \mathrm{~Hz}, 1 \mathrm{H}), 3.47(\mathrm{~d}, J=2.5 \mathrm{~Hz}, 1 \mathrm{H}) .{ }^{13} \mathrm{C}$ NMR $(101$ $\left.\mathrm{MHz}, \mathrm{DMSO}-d_{6}\right) \delta 149.28,142.39,140.29,135.23,134.14,131.78$, $130.75,130.04,128.82,128.76,126.69,124.10,118.31,117.10$, 88.14. HRMS (ESI) $m / z$ : calc. for $\mathrm{C}_{15} \mathrm{H}_{10} \mathrm{Cl}_{2} \mathrm{~N}_{2} \mathrm{O}[\mathrm{M}+\mathrm{H}]^{+}$: 305.0243 , found 305.0229 . 
6-Chloro-4-methylene-3-(m-tolyl)-3,4-dihydroquinazolin2(1H)-one (7v)

White powder. m.p. 201.7 202. $4^{\circ} \mathrm{C} ;{ }^{1} \mathrm{H}$ NMR (400 MHz, DMSO- $\left.d_{6}\right) \delta 10.45(\mathrm{~s}, 1 \mathrm{H}), 7.74(\mathrm{~d}, J=2.3 \mathrm{~Hz}, 1 \mathrm{H}), 7.45-7.32$ $(\mathrm{m}, 2 \mathrm{H}), 7.24(\mathrm{~d}, J=7.7 \mathrm{~Hz}, 1 \mathrm{H}), 7.11-7.02(\mathrm{~m}, 2 \mathrm{H}), 6.96(\mathrm{~d}, J=$ $8.6 \mathrm{~Hz}, 1 \mathrm{H}), 4.88(\mathrm{~d}, J=2.2 \mathrm{~Hz}, 1 \mathrm{H}), 3.49(\mathrm{~d}, J=2.2 \mathrm{~Hz}, 1 \mathrm{H}), 2.35$ $(\mathrm{s}, 3 \mathrm{H}) .{ }^{13} \mathrm{C}$ NMR $\left(101 \mathrm{MHz}, \mathrm{DMSO}-d_{6}\right) \delta 149.44,142.53,139.70$, $138.79,135.35,130.63,130.08,129.97,129.17,126.63,126.57$, $124.03,118.34,117.02,87.93,21.23$. HRMS (ESI) $\mathrm{m} / z$ : calc. for $\mathrm{C}_{16} \mathrm{H}_{13} \mathrm{ClN}_{2} \mathrm{O}[\mathrm{M}+\mathrm{H}]^{+}: 287.0795$, found 287.0802 .

\section{6-Chloro-4-methylene-3-(o-tolyl)-3,4-dihydroquinazolin-} 2(1H)-one $(7 w)$

White powder. m.p. 221.2 222.9 ${ }^{\circ} \mathrm{C} ;{ }^{1} \mathrm{H}$ NMR $(400 \mathrm{MHz}$, DMSO- $\left.d_{6}\right) \delta 10.45(\mathrm{~s}, 1 \mathrm{H}), 7.79(\mathrm{~d}, J=2.3 \mathrm{~Hz}, 1 \mathrm{H}), 7.42-7.32$ (m, 5H), 7.20-7.15 (m, 1H), $6.96(\mathrm{~d}, J=8.6 \mathrm{~Hz}, 1 \mathrm{H}), 4.88(\mathrm{~d}, J$ $=2.1 \mathrm{~Hz}, 1 \mathrm{H}), 3.36(\mathrm{~d}, J=2.2 \mathrm{~Hz}, 2 \mathrm{H}), 2.10(\mathrm{~s}, 3 \mathrm{H}) .{ }^{13} \mathrm{C} \mathrm{NMR}$ $\left(101 \mathrm{MHz}, \mathrm{DMSO}-d_{6}\right) \delta 148.99,141.27,137.48,136.50,135.39$, $131.55,130.75,129.71,128.89,127.95,126.65,124.17,118.06$, $117.10,86.86,17.15$. HRMS (ESI) $m / z$ : calc. for $\mathrm{C}_{16} \mathrm{H}_{13} \mathrm{FN}_{2} \mathrm{O}[\mathrm{M}$ $+\mathrm{H}]^{+}: 285.0789$, found 285.0762 .

\section{3-(3-Chlorophenyl)-7-methyl-4-methylene-3,4- dihydroquinazolin-2(1H)-one (7x)}

White powder. m.p. $214.8 \sim 215.9^{\circ} \mathrm{C} ;{ }^{1} \mathrm{H}$ NMR $(400 \mathrm{MHz}$, DMSO- $\left.d_{6}\right) \delta 10.34(\mathrm{~s}, 1 \mathrm{H}), 7.60-7.48(\mathrm{~m}, 3 \mathrm{H}), 7.45-7.39(\mathrm{~m}, 1 \mathrm{H})$, $7.27(\mathrm{dt}, J=7.7,1.7 \mathrm{~Hz}, 1 \mathrm{H}), 6.84(\mathrm{dd}, J=8.2,1.7 \mathrm{~Hz}, 1 \mathrm{H}), 6.75$ $(\mathrm{s}, 1 \mathrm{H}), 4.74(\mathrm{~d}, J=2.0 \mathrm{~Hz}, 1 \mathrm{H}), 3.35(\mathrm{~d}, J=2.0 \mathrm{~Hz}, 1 \mathrm{H}), 2.28(\mathrm{~s}$, $3 \mathrm{H}) .{ }^{13} \mathrm{C}$ NMR $\left(101 \mathrm{MHz}, \mathrm{DMSO}-d_{6}\right) \delta 149.66,143.54,140.75$, $140.54,136.14,134.08,131.70,130.11,128.90,128.63,124.53$, $123.75,115.28,114.02,85.57,21.38$. HRMS (ESI) $\mathrm{m} / z$ : calc. for $\mathrm{C}_{16} \mathrm{H}_{13} \mathrm{ClN}_{2} \mathrm{O}[\mathrm{M}+\mathrm{H}]^{+}: 285.0789$, found 285.0762 .

\section{3-(2-Fluorophenyl)-7-methyl-4-methylene-3,4-}

dihydroquinazolin-2(1H)-one (7y)

White powder. m.p. $224.3 \sim 224.9^{\circ} \mathrm{C} ;{ }^{1} \mathrm{H}$ NMR $(400 \mathrm{MHz}$, DMSO- $\left.d_{6}\right) \delta 10.44(\mathrm{~s}, 1 \mathrm{H}), 7.57(\mathrm{~d}, J=8.1 \mathrm{~Hz}, 1 \mathrm{H}), 7.55-7.48$ (m, 1H), 7.45-7.39 (m, 2H), 7.35 (ddd, $J=7.9,7.2,1.4 \mathrm{~Hz}, 1 \mathrm{H})$, $4.75(\mathrm{~d}, J=2.1 \mathrm{~Hz}, 1 \mathrm{H}), 3.43(\mathrm{~d}, J=2.1 \mathrm{~Hz}, 1 \mathrm{H}), 2.08(\mathrm{~s}, 2 \mathrm{H}) .{ }^{13} \mathrm{C}$ NMR (101 MHz, DMSO-d $) \delta 156.56(\mathrm{~d}, J=247 \mathrm{~Hz}), 147.29$, $140.58,138.80,133.98,129.98,128.81(\mathrm{~d}, J=8.0 \mathrm{~Hz}), 124.08$ (d, $J$ $=13.3 \mathrm{~Hz}), 123.80(\mathrm{~d}, J=3.5 \mathrm{~Hz}), 122.48,121.78,115.02(\mathrm{~d}, J=$ $19.7 \mathrm{~Hz}$ ), 113.29, 111.62, 82.67, 19.26. HRMS (ESI) $\mathrm{m} / z$ : calc. for $\mathrm{C}_{16} \mathrm{H}_{13} \mathrm{FN}_{2} \mathrm{O}[\mathrm{M}+\mathrm{H}]^{+}:$269.1085, found 269.1062 .

7-Methyl-4-methylene-3-(m-tolyl)-3,4-dihydroquinazolin2(1H)-one (7z)

Blackish green powder. m.p. $161.3 \sim 163.6^{\circ} \mathrm{C} ;{ }^{1} \mathrm{H}$ NMR $(400$ $\left.\mathrm{MHz}, \mathrm{DMSO}-d_{6}\right) \delta 10.25(\mathrm{~s}, 1 \mathrm{H}), 7.54(\mathrm{~d}, J=8.1 \mathrm{~Hz}, 1 \mathrm{H}), 7.40(\mathrm{t}$,
$J=7.7 \mathrm{~Hz}, 1 \mathrm{H}), 7.23(\mathrm{~d}, J=7.7 \mathrm{~Hz}, 1 \mathrm{H}), 7.09-7.01(\mathrm{~m}, 2 \mathrm{H}), 6.82$ $(\mathrm{dd}, J=8.2,1.7 \mathrm{~Hz}, 1 \mathrm{H}), 6.74(\mathrm{~s}, 1 \mathrm{H}), 4.69(\mathrm{~d}, J=1.7 \mathrm{~Hz}, 1 \mathrm{H})$, $3.36(\mathrm{~d}, J=1.7 \mathrm{~Hz}, 1 \mathrm{H}), 2.35$ (s, 3H), 2.28 (s, 3H). ${ }^{13} \mathrm{C}$ NMR $(101$ MHz, DMSO- $\left.d_{6}\right) \delta 149.82,143.69,140.61,139.60,139.05,136.26$, $130.18,129.89,129.04,126.73,124.48,123.64,115.19,114.07$, 85.39, 21.37, 21.22. HRMS (ESI) $m / z$ : calc. for $\mathrm{C}_{17} \mathrm{H}_{16} \mathrm{~N}_{2} \mathrm{O}[\mathrm{M}$ $+\mathrm{H}]^{+}: 265.1335$, found 265.1358 .

\section{3-Benzyl-7-methyl-4-methylene-3,4-dihydroquinazolin- 2(1H)-one (7aa)}

White powder. m.p. 236.8 $238.4^{\circ} \mathrm{C} ;{ }^{1} \mathrm{H}$ NMR $(400 \mathrm{MHz}$, DMSO- $\left.d_{6}\right) \delta 10.28(\mathrm{~s}, 1 \mathrm{H}), 7.50(\mathrm{~d}, J=8.2 \mathrm{~Hz}, 1 \mathrm{H}), 7.32(\mathrm{t}, J=$ $7.5 \mathrm{~Hz}, 2 \mathrm{H}), 7.28-7.19(\mathrm{~m}, 3 \mathrm{H}), 6.79(\mathrm{dd}, J=8.2,1.7 \mathrm{~Hz}, 1 \mathrm{H})$, $6.75(\mathrm{~s}, 1 \mathrm{H}), 4.99(\mathrm{~s}, 2 \mathrm{H}), 4.74(\mathrm{~d}, J=2.3 \mathrm{~Hz}, 1 \mathrm{H}), 4.06(\mathrm{~d}, J$ $=2.4 \mathrm{~Hz}, 1 \mathrm{H}), 2.26(\mathrm{~s}, 3 \mathrm{H}) .{ }^{13} \mathrm{C}$ NMR $\left(101 \mathrm{MHz}, \mathrm{DMSO}-d_{6}\right)$ $\delta 150.72,140.41,140.22,137.58,135.99,128.91,127.18,126.78$, 124.36, 123.68, 115.12, 113.94, 84.68, 46.23, 21.33. HRMS (ESI) $m / z$ : calc. for $\mathrm{C}_{17} \mathrm{H}_{16} \mathrm{~N}_{2} \mathrm{O}[\mathrm{M}+\mathrm{H}]^{+}: 265.1335$, found 265.1326 .

\section{3-Benzyl-6-chloro-4-methylene-3,4-dihydroquinazolin- 2(1H)-one (7ab)}

White powder. m.p. 237.2 $238.9^{\circ} \mathrm{C}$; ${ }^{1} \mathrm{H}$ NMR $(400 \mathrm{MHz}$, DMSO- $\left.d_{6}\right) \delta 10.44(\mathrm{~s}, 1 \mathrm{H}), 7.71(\mathrm{~d}, J=2.2 \mathrm{~Hz}, 1 \mathrm{H}), 7.33(\mathrm{dd}, J$ $=8.8,6.8 \mathrm{~Hz}, 3 \mathrm{H}), 7.27-7.21(\mathrm{~m}, 3 \mathrm{H}), 6.95(\mathrm{~d}, J=8.6 \mathrm{~Hz}, 1 \mathrm{H})$, $4.99(\mathrm{~s}, 2 \mathrm{H}), 4.93(\mathrm{~d}, J=2.8 \mathrm{~Hz}, 1 \mathrm{H}), 4.17(\mathrm{~d}, J=2.8 \mathrm{~Hz}, 1 \mathrm{H})$. ${ }^{13} \mathrm{C}$ NMR $\left(101 \mathrm{MHz}, \mathrm{DMSO}-d_{6}\right) \delta 150.34,139.05,137.27,135.09$, $130.48,128.95,127.27,126.82,126.62,123.93,118.24,116.97$, 87.35, 46.33. HRMS (ESI) $m / z$ : calc. for $\mathrm{C}_{16} \mathrm{H}_{13} \mathrm{ClN}_{2} \mathrm{O}[\mathrm{M}+\mathrm{H}]^{+}$: 285.0789 , found 285.0776 .

\section{DATA AVAILABILITY}

All datasets generated for this study are included in the manuscript/Supplementary Files.

\section{AUTHOR CONTRIBUTIONS}

All authors listed have made a substantial, direct and intellectual contribution to the work, and approved it for publication.

\section{SUPPLEMENTARY MATERIAL}

The Supplementary Material for this article can be found online at: https://www.frontiersin.org/articles/10.3389/fchem. 2019.00584/full\#supplementary-material

Supplementary data $\left({ }^{1} \mathrm{H},{ }^{13} \mathrm{C}\right.$ NMR spectrums, computational data, Cartesian coordinates, Crystal Data) associated with this article can be found in the Supporting Information.

\section{REFERENCES}

Awad, M. K., Abdel-Aal, M. F., Atlam, F. M., and Hekal, H. A. (2018). Design, synthesis, molecular modeling, and biological evaluation of novel alpha-aminophosphonates based quinazolinone moiety as potential anticancer agents: DFT, NBO and vibrational studies. J. Mol. Struct. 1173, 128-141. doi: 10.1016/j.molstruc.2018.06.094

Brack, A. (1969). Über 4-Methylen-chinazolone-(2). Eur. Liebigs Ann. Chem. 730, 166-172. doi: 10.1002/jlac.19697300117

Buckner, F. S., Alvarez, X. B., Fan, E., Gillespie, J. B., Hol, W. G. J., Koh, C. Y., et al. (2015). Specific Inhibitors of Methionyl-tRNA Synthetase. U.S. Patent No US2015046357. Washington, DC: World Intellectual Property Organization.

Camacho, M. E., Chayah, M., García, M. E., Fernández-Sáez, N., Arias, F., Gallo, M. A., et al. (2016). Quinazolinones, quinazolinthiones, and quinazolinimines 
as nitric oxide synthase inhibitors: synthetic study and biological evaluation. Arch. Pharm. 349, 638-650. doi: 10.1002/ardp.201600020

Campbell, H. F., Kuhla, D. E., Studt, W. L., Faith, W. C., and Molino, B. F. (1988). Benzodiazinone-Pyridazinone and Hydroxy-Pyrazolyl Compounds, Cardiotonic Compositions Including the Same, and their Uses. U.S. Patent No US4868300. Doylestown, PA: World Intellectual Property Organization.

Dhami, S., Rumbles, G., MacRobert, A. J., and Phillips, D. (1997). Comparative photophysical study of disulfonated aluminum phthalocyanine in unilamellar vesicles and leukemic K562 cells. Photochem. Photobiol. 65, 85-90. doi: 10.1111/j.1751-1097.1997.tb01881.x

Elkholy, A. E., Rizk, S. A., and Rashad, A. M. (2019). Enhancing lubricating oil properties using novel quinazolinone derivatives: DFT study and molecular dynamics simulation. J. Mol. Struct. 1175, 788-796. doi: 10.1016/j.molstruc.2018.08.045

Frisch, M. J., Trucks, G. W., Schlegel, H. B., Scuseria, G. E., Robb, M. A., Cheeseman, J. R., et al. (2016). Gaussian 09, Revision C.01. Wallingford, CT: Gaussian, Inc.

Fukamachi, S., Konishi, H., and Kobayashi, K. (2010). Synthesis of 2(2-dialkylamino-4H-3,1-benzothiazin-4-yl)acetic acid derivatives and 2-(2thioxo-1,2,3,4-tetrahydroquinazolin-4-yl)acetic acid derivatives. SynthesisStuttgart 10, 1593-1598. doi: 10.1002/chin.201039169

Gatadi, S., Lakshmi, T. V., and Nanduri, S. (2019). 4(3H)-Quinazolinone derivatives: promising antibacterial drug leads. Eur. J. Med. Chem. 170, 157-172. doi: 10.1016/j.ejmech.2019.03.018

Gimeno, A., Cuenca, A. B., Medio-Simon, M., and Asensio, G. (2014a). Gold(I)- catalyzed reactions of 1-(ortho-Alkynylaryl)ureas: highly selective heterocyclization and synthesis of mixed N,O-acetals. Adv. Synth. Catal. 356, 229-236. doi: 10.1002/adsc.201300730

Gimeno, A., Cuenca, A. B., Suárez-Pantiga, S., Ramirez de Arellano, C., MedioSimón, M., and Asensio, G. (2014b). Competitive gold-activation modes in terminal alkynes: an experimental and mechanistic study. Chem. Eur. J. 20, 683-688. doi: 10.1002/chem.201304087

Gimeno, A., Medio-Simón, M., Ramirez de Arellano, C., Asensio, G., and Cuenca, A. B. (2010). NHC-stabilized gold(I) complexes: suitable catalysts for 6exo-dig heterocyclization of 1-(o-ethynylaryl)ureas. Org. Lett. 12, 1900-1903. doi: $10.1021 /$ ol100595s

Grimme, S., Antony, J., Ehrlich, S., and Krieg, H. (2010). A consistent and accurate ab initio parametrization of density functional dispersion correction (DFTD) for the 94 elements H-Pu. J. Chem. Phys. 132:154104. doi: 10.1063/1. 3382344

Grimme, S., Ehrlich, S., and Goerigk, L. (2011). Effect of the damping function in dispersion corrected density functional theory. J. Comput. Chem. 32, 1456-1465. doi: 10.1002/jcc.21759

Grombein, C. M., Hu, Q., Rau, S., Zimmer, C., and Hartmann, R. W. (2015). Heteroatom insertion into 3,4-dihydro-1H-quinolin-2-ones leads to potent and selective inhibitors of human and rat aldosterone synthase. Eur. J. Med. Chem. 90, 788-796. doi: 10.1016/j.ejmech.2014.12.022

Hua, L., Yao, Z., Xu, F., and Shen, Q. (2014). Chemoselective reactions under solvent-free conditions: lanthanide-catalyzed syntheses of 2-amino-3,1benzothiazines and 3,4-dihydroquinazoline-2-thiones. RSC Adv. 4, 3113-3120. doi: $10.1039 / \mathrm{C} 3 \mathrm{RA} 44829 \mathrm{~K}$

Huang, C., Fu, Y., Fu, H., Jiang, Y., and Zhao, Y. (2008). Highly efficient coppercatalyzed cascade synthesis of quinazoline and quinazolinone derivatives. Chem. Commun. 47, 6333-6335. doi: 10.1039/b814011a

Iwamura, H., Fujita, T., Koyama, S., Koshimizu, K., and Kumazawa, Z. (1980). Quantitative structure-activity relationship of cytokininactive adenine and urea derivatives. Phytochemistry 19, 1309-1319. doi: 10.1016/S0031-9422(00)82070-1

Khan, I., Ibrar, A., Abbas, N., and Saeed, A. (2014). Recent advances in the structural library of functionalized quinazoline and quinazolinone scaffolds: synthetic approaches and multifarious applications. Eur. J. Med. Chem. 76, 193-244. doi: 10.1016/j.ejmech.2014.02.005

Khan, I., Ibrar, A., Ahmed, W., and Saeed, A. (2015). Synthetic approaches, functionalization and therapeutic potential of quinazoline and quinazolinone skeletons: the advances continue. Eur. J. Med. Chem. 90, 124-169. doi: 10.1016/j.ejmech.2014.10.084
Khan, I., Zaib, S., Batool, S., Abbas, N., Ashraf, Z., Iqbal, J., et al. (2016). Quinazolines and quinazolinones as ubiquitous structural fragments in medicinal chemistry: an update on the development of synthetic methods and pharmacological diversification. Bioorg. Med. Chem. 24, 2361-2381. doi: 10.1016/j.bmc.2016.03.031

Kleine, T., Fröhlich, R., Wibbeling, B., Würthwein, E., U. (2011). Cyclization reactions of 3,4-diazaheptatrienyl metal compounds. pyridines from an anionic analogue of the fischer indole synthesis: experiment and theory. J. Org. Chem. 76, 4591-4599. doi: $10.1021 /$ jo200487v

Kshirsagar, U. A. (2015). Recent developments in the chemistry of quinazolinone alkaloids. Org. Biomol. Chem. 13, 9336-9352. doi: 10.1039/C5O B01379H

Long, S., Resende, D. I. S. P., Kijjoa, A., Silva, A. M. S., Fernandes, R., Xavier, C. P. R., et al. (2019). Synthesis of new proteomimetic quinazolinone alkaloids and evaluation of their neuroprotective and antitumor effects. Molecules 24:534. doi: 10.3390/molecules 24030534

Maiden, T. M., and Harrity, J. P. (2016). Recent developments in transition metal catalysis for quinazolinone synthesis. Org. Biomol. Chem. 14, 8014-8025. doi: $10.1039 /$ С6OB01402J

Markovic, Z. S., Markovic, J. M. D., and Dolicanin, C. B. (2010). Mechanistic pathways for the reaction of quercetin with hydroperoxy radical. Theor. Chem. Acc. 127, 69-80. doi: 10.1007/s00214-009-0706-x

Mei, Q., Wang, L., Tian, B., Yan, F., Zhang, B., Huang, W., et al. (2012). A highly selective and naked-eye sensor for $\mathrm{Hg}^{2+}$ based on quinazoline-4(3H)-thione. New J. Chem. 36, 1879-1883. doi: 10.1039/c2nj 40400a

Molina, P., Conesa, C., Alías, A., Arques, A., Velasco, M. D., Llamas-Saiz, A. L., et al. (1993). Preparation and synthetic applications of iminophosphoranes derived from o-substituted arylazides: preparation of pyrazolo[1,2-b]indazole, $4 \mathrm{H}-3,1$-benzoxazine and quinoline derivatives. Crystal structure of 2-[2-(4methoxybenzoylamino)phenyl]-4-methylq. Cheminformatics 49, 7599-7612. doi: 10.1016/S0040-4020(01)87234-3

Morgenstern, O., and Richter, P. H. (1992). Chemistry and biological-activity of 1,3,4-benzotriazepines. 2. Die Pharm. 47, 655-677.

Saunthwal, R. K., Patel, M., Tiwari, R. K., Parang, K., and Verma, A. K. (2015). On water: catalyst-free chemoselective synthesis of highly functionalized tetrahydroquinazolines from 2aminophenylacrylate. Green Chem. 17, 1434-1441. doi: 10.1039/C4G C02154A

Sawant, R. T., Stevens, M. Y., and Odell, L. R. (2015). Rapid access to polyfunctionalized 3,4-dihydroquinazolinones through a sequential $\mathrm{N}$ acyliminium ion mannich reaction cascade. Eur. J. Med. Chem. 35, 7743-7755. doi: $10.1002 /$ ejoc.201501178

Sbei, N., Batanero, B., Barba, F., Haouas, B., Benkhoud, M. L., and Barba, I. (2018). Facile preparation of 3-substituted 2-quinazolinones via electrogenerated base. Tetrahedron 74, 2068-2072. doi: 10.1016/j.tet.2018. 03.010

Thanigaimalai, P., Sharma, V. K., Lee, K.-C., Yun, C.-Y., Kim, Y., and Jung, S.-H. (2010). Refinement of the pharmacophore of 3,4-dihydroquinazoline$2(1 \mathrm{H})$-thiones for their anti-melanogenesis activity. Bioorg. Med. Chem. Lett. 20, 4771-4773. doi: 10.1016/j.bmcl.2010.06.123

Walser, A., Flynn, T., Mason, C., Crowley, H., Maresca, C., Yaremko, B., et al. (1991). Triazolobenzo- and triazolothienodiazepines as potent antagonists of platelet activating factor. J. Med. Chem. 34, 1209-1221. doi: $10.1021 / \mathrm{jm} 00107 \mathrm{a} 048$

Wang, H., Liu, L., Wang, Y., Peng, C., Zhang, J., and Zhu, Q. (2009). An efficient synthesis of 4-alkyl-2(1H)-quinazolinones and 4-alkyl-2-chloroquinazolines from 1-(2-alkynylphenyl)ureas. Tetrahedron Lett. 50, 6841-6843. doi: 10.1016/j.tetlet.2009. 09.130

Wang, H.-X., Liu, H.-Y., Li, W., Zhang, S., Wu, Z., Li, X., et al. (2019). Design, synthesis, antiproliferative and antibacterial evaluation of quinazolinone derivatives. Med. Chem. Res. 28, 203-214. doi: 10.1007/s00044-018-2276-8

Xie, T., Xiao, Y., Zhao, S., Hu, X.-Q., and Xu, P.-F. (2016). Catalystfree chemoselective synthesis of 3,4-dihydroquinazoline-2-thiones and 2-imino[1,3]benzothiazines. J. Org. Chem. 81, 10499-10505. doi: 10.1021/acs.joc.6b01232 
Yan, H., Hider, R. C., and Ma, Y. (2019). Synthesis, characterisation and quantum chemical studies of a new series of iron chelatable fluorescent sensors. Mol. Phys. 117, 661-671. doi: 10.1080/00268976.2018.1533147

Zeiger, M., Stark, S., Kalden, E., Ackermann, B., Ferner, J., Scheffer, U., et al. (2014). Fragment based search for small molecule inhibitors of HIV-1 Tat-TAR. Bioorg. Med. Chem. Lett. 24, 5576-5580. doi: 10.1016/j.bmcl.2014.11.004

Zhang, Y., Shao, Y., Gong, J., Hu, K., Cheng, T., and Chen, J. (2018). Palladiumcatalyzed tandem reaction of quinazolinone-based nitriles with arylboronic acids: synthesis of 2-(4-arylquinazolin-2-yl)anilines. Adv. Synth. Catal. 360, 3260-3265. doi: 10.1002/adsc. 201800615
Conflict of Interest Statement: The authors declare that the research was conducted in the absence of any commercial or financial relationships that could be construed as a potential conflict of interest.

Copyright (C) 2019 Yan, Xiao, Hider and Ma. This is an open-access article distributed under the terms of the Creative Commons Attribution License (CC BY). The use, distribution or reproduction in other forums is permitted, provided the original author(s) and the copyright owner(s) are credited and that the original publication in this journal is cited, in accordance with accepted academic practice. No use, distribution or reproduction is permitted which does not comply with these terms. 